\title{
Kinetics and Dynamics of Phase Transformations in Metals Under Action of Ultra-Short High-Power Laser Pulses
}

\author{
V.I. Mazhukin \\ Additional information is available at the end of the chapter
}

http://dx.doi.org/10.5772/50731

\section{Introduction}

Action of super-power and ultra-short laser pulses on highly absorbing condensed media is investigated in the past two decades [1] - [5]. The urgency of this problem is primarily determined by a variety of practical applications of pulsed laser irradiation. In a short period of time scientists have mastered such operations as ablation of elemental materials by femtosecond lasers (100 fs) [6], femtosecond nanostructuring [7], generation of metallic nanoparticles and nanostructures by laser ablation of massive targets by $40 \mathrm{fs}$ and $2 \mathrm{ps}$ pulses [8], etc.

The use of super-power $\mathrm{G} \sim 10^{12} \div 10^{15} \mathrm{~W} / \mathrm{cm}^{2}$ and ultra-short $\tau_{\mathrm{L}} \approx 10^{-12} \div 10^{-15} \mathrm{~s}$ laser pulses for dimensional processing of materials, such as cutting of various materials by picofemtosecond laser pulses [9], micro-drilling by a femtosecond laser [10 ], surface etching of metals ( $\mathrm{Al}, \mathrm{Cu}, \mathrm{Mo}, \mathrm{Ni}$ ) and semiconductors (Si) [11], is accompanied by realization of unique physical conditions. In particular, the duration of action becomes comparable with the characteristic times of thermalization and phase transitions in matter. This leads to the need to address complex fundamental problems, including the heating of the material and the kinetics of phase transitions in a strong deviation from local thermodynamic equilibrium.

It should be noted that the majority of laser technologies is associated with the beginning of phase transformations in the material. In particular, the action of pico - femtosecond laser pulses of high intensity on solid targets is one of the ways to create individual particles with unique characteristics, or to form their streams, consisting of a cluster, liquid or solid fragments of the target. The formation of the particle flux in the pulsed laser ablation is observed for a wide range of materials: metals, semiconductors and insulators. The 
possibility of usage of laser ablation products in practical applications was the impetus for a number of experimental and theoretical studies [12], aimed at studying the conditions and mechanisms of formation of particles of nano - and micro- sizes during the laser exposure.

The accumulation of knowledge of the experimental nature in the first place in this dynamic field leads not only to the variety of practical applications of pulsed laser action, but also to a number of issues of independent physical interest.

The main features of ultrashort action on metals are associated with high speed and voluminous nature of the energy release of the laser pulse. The high rate of heating of a condensed medium is associated with rapid phase transformations of matter, characterized by the transfer of superheated phase boundaries of high-power fluxes of mass and energy. Overheated metastable states at the interface are characterized by temperatures, whose values can be hundreds of degrees higher than the equilibrium values of the melting point or boiling point. Removal of the energy by the flow of matter in conjunction with volume mechanism of energy release of laser radiation contribute to the formation of metastable superheated regions in the volume of solid and liquid phases with near-surface temperature maximum. Calculations [13] showed that the maximum speed of melting front are comparable to the speed of sound $\sim(0.5-6) \mathrm{km} / \mathrm{s}$, and the phase velocity of the solidification front are $(10-200) \mathrm{m} / \mathrm{s}$. Accordingly, the maximum superheating / undercooling can reach several thousand / hundred degrees. The achievement of such overheating and overcooling leads to large gradients of the Gibbs energy, which actually determine the driving force for high-speed phase transformations.

Physics of supercooled states in metallic and nonmetallic systems, because of their widespread use in the production of new materials technology, is relatively well-studied [14]. Superheated states received little attention until recently, largely because of the difficulty of experimental investigation and non-obviousness of their application. The situation changed with the advent of femtosecond laser pulses and their application for the production of nanoparticles and nanomaterials [15] - [17].

The purpose of this chapter is the theoretical study of nonequilibrium pulsed laser heating of metals, the kinetics and dynamics of phase transitions in a deep deviation from the local thermodynamic equilibrium.

The main tool for studying the processes initiated by laser pulses of picosecond and femtosecond duration are the methods of mathematical modeling and computational experiment (CE). The possibilities of experimental approaches in this area are very limited due to the large transience of the processes. Computational experiments are preferred in the cases where the natural experiment is not possible, is very difficult or very expensive. The statement of CE is especially convincing in the studies of the kinetics and dynamics of fast processes. For its statement, the computational experiment, which is an important link connecting theory and field experiments, requires the development of appropriate models, determining the properties of all substances studied, the development of computational algorithms and the creation of program codes. 
Construction of a theory that covers most of the features of pulsed laser action on materials is very difficult. The methods of mathematical modeling in theoretical constructs have that advantage that you can use the phenomenological and the experimental data.

The description of the kinetics of fast phase transitions of the first kind is carried out in two classes of mathematical models: continuum and atomistic. Continuous models are based on the equations of continuum mechanics, as a rule, are represented as partial differential equations with appropriate boundary conditions and equations of state. Continuum model is used to describe the macro-level processes, heterogeneous kinetics and dynamics of phase transitions of the 1st kind [18]. Atomistic approach is based on a model of molecular dynamics is used to describe the kinetics of homogeneous phase transitions [19].

\section{Brief theory of phase transformations of the $1^{\text {st }}$ type}

The basis of first-order phase transformations - melting-solidification and evaporationcondensation are two qualitatively different mechanisms: heterogeneous and homogeneous ones. The heterogeneous mechanism is characterized by a sharp interface between the phases (phase front of zero thickness) and determines the dynamics of phase transformations of the 1st type. The homogeneous mechanism of phase transitions that arise usually under the influence of the volume heating or cooling is associated with the processes of volume melting and boiling or spontaneous crystallization and condensation.

Historically, the theory of phase transformations of the 1st type of melting is based on two fundamental sections of classical physics, thermodynamics and kinetics.

\subsection{Thermodynamical approach}

Thermodynamics is a macroscopic theory [20], which from the energy point of view, considers the properties of macroscopic bodies in equilibrium. This allows to have a great community for the conclusions of thermodynamics. However, thermodynamics does not take into account the internal structure of the considered bodies and some of its conclusions and regulations do not have physical clarity. One way to describe the equilibrium processes in the equilibrium thermodynamics is a theory of thermodynamic potentials.

\subsubsection{Phase equilibrium and phase transformations}

The properties of a thermodynamic system are determined by thermodynamic parameters. The energy state of a thermodynamic system in equilibrium is uniquely determined by the parameters of the system. There is a unique relationship between the parameters of the system, which is mathematically represented by the state function. From a mathematical point of view, this means that the function has a total differential.

The basis of the method of thermodynamic potentials is just the possibility of introducing the state functions for the equilibrium processes, with total differentials describing the change in the state of a thermodynamic system. 
The main identity of thermodynamics of equilibrium processes is usually represented as

$$
T d S=d \varepsilon+p d V \text { or } d \varepsilon=T d S-p d V
$$

Depending on the choice of the two independent parameters, one can introduce thermodynamic potentials, which differentiation allows determining the other unknown parameters of the state. In general, a thermodynamic potential may be a function of various parameters. In this notation, the internal energy is given as a function of entropy and volume: $\varepsilon=\varepsilon(S, V)$. It is the function of the state and it has a total differential with respect to its variables. The total differential $d \varepsilon$ can be used to determine the temperature $T$ and pressure $p$. However, the usage of entropy $S$ and volume $V$ as two independent variables is inconvenient because they are difficult to control in experiment. It is more convenient to use pressure $p$ and temperature $T$ as two independent variables.

After consecutive transformation of internal energy $U$ first into enthalpy $H=H(S, p)$

$$
H=\varepsilon+p V
$$

which total differential, taking into account the basic identity of thermodynamics has the form

$$
d H=d \varepsilon+p d V+V d p=T d S+V d p
$$

and then into Gibbs energy $G=G(T, p)$ (Gibbs thermodynamic potential)

$$
G=H-T S=\varepsilon+p V-T S
$$

one can obtain the total differential of Gibbs energy, which with account of the basic identity of thermodynamics (1), takes the form

$$
d G=-S d T+V d p
$$

It is convenient in the fact that the independent variables $T$ and $p$ are easily to modify and to control in experiment.

After reaching the equilibrium state of the system, Gibbs potential takes its minimum value and becomes constant: $d G=0$. This allows to use the condition of minimum of the Gibbs potential for the description of equilibrium states in which $T=$ const and $p=$ const .

Thermodynamic systems in equilibrium state do not necessarily have to be a homogeneous medium. A system in equilibrium may be composed of several phases, different in their physical and chemical properties, separated by the phase boundaries not changing over time.

The multiphase thermodynamic systems are most simply described, components of which are in equilibrium states, and there is no transfer of matter, energy and momentum through interphase boundaries. In this case, such thermodynamic system is in equilibrium and methods of equilibrium thermodynamics apply to describe it. 
Given that during phase transformations, each of the phases is a system with variable mass, the notion of chemical potential $\mu(p, T)$ is introduced into the thermodynamic description. It is used to take into account not only exchange of energy but also the exchange of mass (particles). To determine the chemical potential, the term which takes into account the possibility of changing the number of particles in a homogeneous system (the same can be done with the other potentials) is formally introduced into the expression for the thermodynamic Gibbs potential:

$$
d G=-S d T+V d p+\mu d N
$$

$d N$ determines the change of the number of particles in the system.

If the thermodynamic potential $d G$ is given as a function of temperature and pressure, then the value of the chemical potential is written as: $\mu=\left(\frac{\partial G}{\partial N}\right)_{p, T}$

The chemical potential can be written through other thermodynamical functions but in this case it will be written in terms of other state parameters:

$$
\mu=\left(\frac{\partial G}{\partial N}\right)_{p, T}=\left(\frac{\partial U}{\partial N}\right)_{V, S}=\left(\frac{\partial H}{\partial N}\right)_{p, S}
$$

From the relation (6) it follows, that the chemical potential is a physical quantity that is equal to the value of some thermodynamical potential (with constant certain parameters) that is required to add to the system to change its number of particles by unity.

\subsubsection{The conditions of equilibrium for two-phase single-component system}

If macroscopic transport does not occur through the interphase boundaries, and the phases themselves are in a state of thermodynamic equilibrium, such thermodynamic system, in spite of its heterogeneity, will be in a state of thermodynamic equilibrium.

For the phase equilibrium in the one-component two-phase system, the following three conditions must be fulfilled:

1. the condition of thermal equilibrium, that means the equality of temperatures at both sides of the interphase boundary: $T_{1}=T_{2}=T_{\text {eq }}$,

2. the condition of mechanical equilibrium, consisting of the equality of pressure at the both sides of the interphase boundary: $p_{1}=p_{2}=p_{e q}$,

3. the condition of the equality of the Gibbs energy per particle, consisting of the requirement of the absence of the macroscopic transfer of molecules (atoms) of this material from one phase to another: $\mu_{1}(p, T)=\mu_{2}(p, T)$

In principle, we can use not the Gibbs energy, but any thermodynamic potential, which has a minimum in equilibrium. It is not difficult to show the validity of these conditions. We 
shall use the total differential for the internal energy, taking into account changes in the number of particles:

$$
d \varepsilon=T d S-p d V+\mu d N
$$

We write this expression for each phase of the closed-loop system:

$$
\begin{gathered}
d \varepsilon_{1}=T_{1} d S_{1}-p_{1} d V_{1}+\mu_{1} d N_{1} \\
d \varepsilon_{2}=T_{2} d S_{2}-p_{2} d V_{2}+\mu_{2} d N_{2}
\end{gathered}
$$

The closedness of the system automatically gives the following equation:

$$
d \varepsilon_{1}=d \varepsilon_{2}, \quad d S_{1}=d S_{2}, \quad d V_{1}=-d V_{2}, \quad d N_{1}=-d N_{2} .
$$

Consider an equilibrium two-phase system under some simplifying assumptions. Assume that the phases do not change the volume and do not exchange particles, i.e. $d V_{1}=d V_{2}=0$, $d N_{1}=d N_{2}=0$. Combining the equations (7) for this case, we obtain the expression: $d \varepsilon_{1}+d \varepsilon_{2}=d S_{1}\left(T_{1}-T_{2}\right)=0$, which gives the condition of thermal equilibrium $T_{1}=T_{2}=T_{e q}$. Assuming the constancy of the entropy and the number of particles in phases, i.e. $d S_{1}=d S_{2}=0, d N_{1}=d N_{2}=0$ we obtain the condition of mechanical equilibrium: $p_{1}=p_{2}=p_{e q}$.

Given the conditions of thermal and mechanical equilibrium of (7) we obtain the equality of chemical potentials in the different phases $\mu_{1}(p, T)=\mu_{2}(p, T)$. This equation can be solved for the variables $T$ and $p$ and may represent the equilibrium curves of the two phases in the form $p_{e q}=p\left(T_{e q}\right)$ or $T_{e q}=T\left(p_{e q}\right)$. If we consider the boundary between liquid and solid, the equilibrium melting curve is obtained $T_{m}=T\left(p_{m}\right)$.

When describing the interface between liquid and gas, the equilibrium vaporization curve is obtained $p_{\text {sat }}=p\left(T_{b}\right)$.

It should be noted that the processes at the interface are static in nature as in the case of equilibrium of different phases and also during phase transitions. There is a constant process of transition of particles from one phase to another at the interface. In equilibrium, these opposing processes compensate each other, and during supply or withdrawal of heat to one of the phases one of these processes begins to dominate leading to a change in the amount of matter in various states of aggregation.

If the components of the thermodynamic system are not in equilibrium with each other, then there are thermodynamic flows through their interface. This will be a process of transformation of matter from one state to another, i.e. phase transformation. Assuming that the occurring processes are quasi-static and the flows are small, one can use the methods of equilibrium thermodynamics to describe such non-equilibrium system. In this case we assume an infinitely small difference between the thermodynamic parameters in different parts of the system. 


\subsubsection{The driving force of phase transformations}

In the process of phase transitions of the 1st type, a number of quantities undergo abrupt changes at the interface, so in the following text in the thermodynamic equations, the sign of the differential will be replace by the corresponding value of the difference $d F \approx \Delta F$ for the temperature of the phase transition $T_{e q}$.

The driving force of phase transitions of the first type is determined by the difference of Gibbs energy (or the magnitude of overheating/overcooling $\Delta T$ ) for two phases at the interface, defined in (4) and (5) and can be written in two forms

$$
\begin{gathered}
\Delta G=\left(\varepsilon_{1}-\varepsilon_{2}\right)+p_{e q}\left(V_{1}-V_{2}\right)-T_{e q}\left(S_{1}-S_{2}\right)=\Delta \varepsilon+p_{e q} \Delta V-T_{e q} \Delta S \\
\Delta G=V \Delta p-S \Delta T
\end{gathered}
$$

where $\Delta p=p-p_{e q}, \quad \Delta T=T-T_{e q}, \quad \Delta \varepsilon=\varepsilon_{1}-\varepsilon_{2}, \quad \Delta V=V_{1}-V_{2}, \quad \Delta S=S_{1}-S_{2}$

Equilibrium. In equilibrium, $\Delta G=0$ and the equality (8) takes the form:

$$
\Delta \varepsilon+p_{e q} \Delta V-T_{e q} \Delta S=\Delta H-T_{e q} \Delta S=0
$$

where the difference of enthalpy $\Delta H=\Delta \varepsilon+p_{e q} \Delta V$, is known as the equilibrium latent heat of transformation

$$
L_{e q}=\Delta H=T_{e q} \Delta S .
$$

From equation (9) we can obtain the dependence of the equilibrium pressure $P_{e q}$ on the temperature $T_{e q}$, that is known as the curve of Clausius-Clapeyron:

$$
\frac{d p_{e q}}{d T_{e q}}=\frac{L_{e q}}{T_{e q}} \frac{1}{\Delta V}
$$

If one take as $\Delta V$ the difference of the volumes of vapor $V_{\text {vap }}$ and condensed $V_{\text {sol }}$ phases, then since $V_{\text {vap }} \gg V_{\text {sol }}$ for ideal gas $\Delta V=V_{\text {vapor }}-V_{\text {solid }} \approx \frac{R T}{P}$ one obtain the expression

$$
\frac{d p_{e q}}{p_{e q}}=\frac{L_{v}}{R} \frac{d T_{e q}}{T_{e q}^{2}}
$$

After integration, we find the temperature dependence of the equilibrium vapor pressure that is widely used for many materials:

$$
p_{e q}=p_{b} \exp \left[\frac{L_{v}}{R}\left(\frac{1}{T_{b}}-\frac{1}{T_{e q}}\right)\right]
$$


The vapor pressure of the material in equilibrium with solid or liquid phase is called the saturated vapor pressure and with the notation $p_{e q}=p_{\text {sat }}, T_{e q}=T_{\text {sur }}$ is usually written as

$$
p_{\text {sat }}=p_{b} \exp \left[\frac{L_{v}}{R}\left(\frac{1}{T_{b}}-\frac{1}{T_{\text {sur }}}\right)\right]
$$

where $T_{\text {sur }}$ is the temperature of the surface of the condensed phase, $p_{b}, T_{b}$ are the equilibrium values of pressure and the boiling point under normal conditions, $L_{v}$ is the latent heat of evaporation, $R$ is the gas constant.

As it follows from equation (9), at constant pressure, the difference of Gibbs energy $\Delta G$ is linearly proportional to the overheating/ overcooling

$$
\Delta G=-S \cdot \Delta T=\frac{L_{e q} \Delta T}{T_{e q}}
$$

In the future, assuming the difference of energy $\Delta G$ to be equal to the rate of the phase transformation, one can obtain, that in the thermodynamic approach, the rate of conversion at constant pressure for small deviations from equilibrium is linearly proportional to the overheating / overcooling $\Delta \mathrm{T}$

$$
v \approx K \Delta T
$$

where $K$ is the constant of proportionality between the normal speed limits and its overcooling. The constant $K$ does not have any clear physical sense and is chosen experimentally for each material. The dependence (14) by its form coincides with the well known relation for the determination of the linear crystal growth rate obtained on the basis of classical molecular-kinetic models in which the constant of proportionality is called the kinetic coefficient [21]. The main application of the relation (14) and its various modification $[22,23]$ is found in the description of different processes of melting - solidification. Comparison with experiment showed that the equation (14) gives good agreement mainly at small overcoolings $[24,25]$. The kinetic coefficient $K$ is the main parameter, characterizing the mobility of the boundary crystal -melt. Despite the great importance of this characteristic, there are only a few experiments to successfully measure the kinetic coefficient in metals and alloys [26]. The main difficulties of experimental determination are associated with the great complexity of measuring the overcooling at the solidification front. Currently, the main approach to the determination of the quantitative evaluation and qualitative understanding of the physics of the processes behind the coefficient $K$ are the methods of molecular dynamics [27] - [30].

\subsection{Kinetic approach}

The structure particles of matter are in continuous motion and appear in the main provisions of the molecular-kinetic theory in which all processes are considered at the 
atomic or molecular level, the particles obey the Boltzmann statistics, and the speed of processes is given by

$$
R=R_{0} \exp \left(-Q / k_{B} T\right)
$$

where $Q$ is the activation energy, $k_{B}$ is the Boltzmann's constant, $k_{B} T$ is the average thermal energy for one atom, $R_{0}$ is the pre-exponential factor that affects the process rate. The exponential term $\exp \left(-Q / k_{B} T\right)$ is known as Boltzmann's factor, that determines the part of atoms or molecules in the system that have the energy above $Q$ at the temperature $T$.

\subsubsection{Melt kinetics}

For the first time, the conditions of crystal growth from liquids were formulated by Wilson [31], who suggested that the atoms have to overcome the diffusion barrier in order to make the transition from liquid to solid phase. The rate of accession of atoms to the crystal lattice is expressed by the relation analogous to (15)

$$
R_{c}(T)=a v \exp \left(-Q / k_{B} T\right)
$$

where $Q$ is the activation energy for overcoming the diffusion barrier, $v$ is the frequency of attempts of transitions, $a$ is the atom diameter.

The rate of accession of atoms to the crystal was estimated in [31] using the diffusion coefficient of liquid $D=\frac{a^{2}}{6} v_{D} \exp \left(-Q / k_{B} T\right)$, that allowed to estimate the velocity of the crystallization front as

$$
v_{s \ell}=\frac{6 D}{a} \frac{L_{m}}{k_{B} T_{m}} \frac{\Delta T}{T_{s \ell}}
$$

where $\Delta T=T_{s \ell}-T_{m}, T_{s \ell}$ is the temperature of the interphase boundary.

Later, Frenkel [32], using the Stokes-Einstein relation $D=\frac{k_{B} T}{3 \pi a \eta}$ between the coefficients of diffusion $D$ and viscosity $\eta$ expressed the crystal growth rate in terms of viscosity

$$
v_{s \ell}=\frac{2 k_{B} T}{\pi a^{2} \eta} \frac{L_{m}}{R T_{m}} \frac{\Delta T}{T_{s \ell}}
$$

The expressions (17), (18) allowed establishing a linear relationship between the rates of crystal growth and overcooling. Wilson-Frenkel theory [31-33] contributed to a better understanding of the microscopic processes associated with the growth of crystals from the melt. A generalization of the obtained results allowed to obtain that, except for very large deviations from equilibrium, where the homogeneous nucleation mechanism can dominate, 
the process of melting-solidification proceeds heterogeneously. The heterogeneous nucleation mechanism involves the inclusion of the motion of the liquid-solid interface into the consideration. The velocity of this interface $v_{s \ell}$, as a function of the deviation from the equilibrium melting temperature $T_{m}$ is called the response function of the interface and is the main value characterizing the processes of crystallization and melting. As with the similar equation (14) obtained in the thermodynamic approach, the equations of WilsonFrenkel showed a good agreement with experiments for very small overcooling of the boundary, i.e. for small deviations from equilibrium.

Theoretical studies of the velocity of the interface are based on some modifications and generalizations of Wilson-Frenkel theory. Their meaning is reduced to taking into account several factors, such as the latent heat of melting $L_{m}$, interatomic distance $a$, efficiency coefficient $f$, showing the proportion of atoms that remain in the solid phase at the border crossing. In the modifications [24,34,35], it was considered that the crystal has always lower enthalpy than the melt. This is the amount of energy needed for atoms of the crystal to make the transition from crystal to melt. Escape rate of atoms of the crystal followed by the addition to the active points of the liquid, contains this energy difference in the form of the Boltzmann factor $\exp \left(-L_{m} / k_{B} T_{s \ell}\right)$

$$
R_{m}\left(T_{s \ell}\right)=C_{1} \exp \left(-Q / k_{B} T_{s \ell}\right) \exp \left(-L_{m} / k_{B} T_{s \ell}\right)
$$

The rate of the reverse flow of atoms into the crystal from the melt depends only on the diffusion process in liquid

$$
R_{c}\left(T_{s \ell}\right)=C_{2} \exp \left(-Q / k_{B} T_{s \ell}\right)
$$

where $C_{1}, C_{2}$ are some constants that should be determined.

In the equilibrium point $T_{s \ell}=T_{m}$, the rates $R_{m}\left(T_{m}\right)$ and $R_{c}\left(T_{m}\right)$ are equal, which gives $C_{1} \exp \left(-Q / k_{B} T_{m}\right) \exp \left(-L_{m} / k_{B} T_{m}\right)=C_{2} \exp \left(-Q / k_{B} T_{m}\right)$, and $C_{1}=C_{2} \exp \left(L_{m} / k_{B} T_{m}\right)$.

The velocity of the interphase boundary $v_{s \ell}$ is equal to the difference of the rates $R_{c}(T)$ и $R_{m}(T)$

$$
\begin{aligned}
& v_{s \ell}\left(T_{s \ell}\right)=R_{c}\left(T_{s \ell}\right)-R_{m}\left(T_{s \ell}\right)=C_{2} \exp \left(-Q / k_{B} T_{s \ell}\right)-C_{1} \exp \left(-Q / k_{B} T_{s \ell}\right) \exp \left(-L_{m} / k_{B} T_{s \ell}\right)= \\
= & \exp \left(-Q / k_{B} T_{s \ell}\right)\left[C_{2}-C_{1} \exp \left(-L_{m} / k_{B} T_{s \ell}\right)\right]=C_{2} \exp \left(-Q / k_{B} T_{s \ell}\right)\left[1-\exp \left(\frac{L_{m}}{k_{B}}\left(\frac{1}{T_{m}}-\frac{1}{T_{s \ell}}\right)\right)\right]= \\
= & C_{2} \exp \left(-Q / k_{B} T_{s \ell}\right)\left[1-\exp \left(\frac{L_{m}}{k_{B}}\left(\frac{T_{s \ell}-T_{m}}{T_{s \ell}}\right)\right)\right]=C_{2} \exp \left(-Q / k_{B} T_{s \ell}\right)\left[1-\exp \left(\frac{L_{m}}{k_{B}}\left(\frac{\Delta T}{T_{s \ell}}\right)\right)\right]
\end{aligned}
$$

$\Delta T=T_{s \ell}-T_{m}$, for overheating $\Delta T>0$, for overcooling $\Delta T<0$.

The constant $C_{2}$ is associated with other physical constants using the following relation: $C_{2}=a f v$. The studies have shown that the resulting equation (21) well predicts the velocity of the melting-solidification front of silicon [36] in a fairly wide temperature range. 
The problems of melting-crystallization of monatomic metals in the modes of rapid heating/ cooling, typical of ultra-short laser irradiation, use a different expression for the velocity $v_{s \ell}$. It is based on the assumption [37, 38], that crystallization of single-atom metals, (that are characterized by high velocities $v_{s \ell} \approx 50 \div 100 \mathrm{~m} / \mathrm{c}[35,39]$ ) is not diffusion-limited, but is limited only by the collision frequency during transition from liquid to crystal surface. The modification of the equation (21) consists of replacement of the diffusion term with the thermal velocity $v_{T}=\left(3 k_{B} T / m\right)^{1 / 2}$. The velocity of interphase boundary $v_{s \ell}$ is written as:

$$
v_{s \ell}\left(T_{s \ell}\right)=\frac{a f}{\lambda}\left(3 k_{B} T_{s \ell} / m\right)^{1 / 2}\left[1-\exp \left(\frac{L_{m}}{k_{B}}\left(\frac{\Delta T}{T_{s \ell}}\right)\right)\right]=C \cdot\left(T_{s \ell}\right)^{1 / 2}\left[1-\exp \left(\frac{L_{m}}{k_{B}}\left(\frac{\Delta T}{T_{s \ell}}\right)\right)\right]
$$

where $C=\frac{a f}{\lambda}\left(3 k_{B} / m\right)^{1 / 2}, \lambda$ is the mean free path, $m$ is the atomic mass.

Thus, the kinetic approach allows us to obtain an expression for the response function $v_{s \ell}\left(T_{s \ell}\right)$ without fitting coefficients and suitable for a wide range of overheating/overcooling. The kinetic dependence (22) is asymmetric for the processes of melting and solidification. However, large deviations from equilibrium require additional modification of the kinetic dependences (21) (22), because they do not take into account the dynamic effects associated with the occurrence of high pressures generated by the high velocity of propagation of phase fronts.

\subsubsection{Kinetics of vapor}

One of the key processes in the zone of laser irradiation is the transition of condensed matter to the gaseous state. Evaporation process is characterized by high power consumption and large increase in the specific volume of the substance.

Investigation of evaporation process began in the $19^{\text {th }}$ century $[40,41]$ and continues to this day [42 -]. This fact is defined by practical importance and not fully clarified features of nonequilibrium behavior of matter when it evaporates.

\subsubsection{The simplest model of kinetics of evaporation in vacuum}

The thermodynamic relation (9) gives that at a constant temperature the difference between the Gibbs energy between the two phases, one of which is an ideal gas, is linearly proportional to the pressure difference:

$$
\Delta G=V \Delta p=k_{B} T \frac{\Delta p}{p}
$$

On the basis of the formula (23), it can be assumed that the rate of phase transformation at constant temperature should be linearly proportional to the pressure difference:

$$
v=v_{0} \frac{\Delta p}{p}
$$


The simplest model for the growth kinetics of vapor phase was developed by Hertz [40] and Knudsen [41] about 100 years ago. Its formulation is qualitatively the same as the thermodynamic model (23), (24)

$$
j_{m}=j_{m}^{+}-j_{m}^{-}=\frac{1}{\sqrt{2 \pi m k_{B}}}\left(\frac{p_{\text {sat }}}{\sqrt{T_{\text {sur }}}}-\frac{p}{\sqrt{T}}\right)
$$

where $j_{m}$ is non-equilibrium flow of atoms at the surface of evaporation, $j_{m}^{-}$is the flux of atoms that collide with the surface under the assumption that the adhesion coefficient is 1 . Formally, this flow can be determined using the relation connecting the vapor pressure with the equilibrium particle flux directed to the condensed surface: $p=\sqrt{2 \pi} m v j_{m}^{-}$, where $v$ is the average velocity in one direction, $v=\sqrt{\frac{2 k_{B} T}{m}}$, what gives $j_{m}^{-}=\frac{p}{\sqrt{2 \pi m k_{B} T}}$. Since the nature of the flux $j_{m}^{(-)}$remained undetermined and the values of $T$ and correspondingly $p$ unknown, then during the formulation of the boundary conditions in the problem of evaporation into vacuum it is supposed [20, p.281] to use a single-term version of the HertzKnudsen formula to determine the evaporation rate:

$$
j_{m} \approx j_{m}^{+}=\frac{p_{\text {sat }}}{\sqrt{2 \pi m k_{B} T_{\text {sur }}}}
$$

that takes into account the connection between the pressure of saturated vapor $p_{\text {sat }}$ and flux of evaporated atoms at the surface temperature $T_{\text {sur }}$. The equilibrium vapor pressure $p_{\text {sat }}$ depends on the temperature $T_{\text {sur }}$, as it is shown in the equation (12).

However, the representation of the process of surface evaporation in the form of a simple model, which does not take into account the reverse influence of evaporated atoms, does not remove the internal contradictions inherent in the model of Hertz - Knudsen. Let us write the expressions for the fluxes of momentum and $j_{i}$ and energy $j_{e}$ of the particles, moving away from the evaporation surface

$$
\begin{gathered}
\rho<V_{z}^{2}>=j_{i}^{(+)}+j_{i}^{(-)}=j_{i}=\rho_{v} R T_{v}+\rho_{v} u^{2} \\
\rho<V^{2} V_{z}>=j_{e}^{(+)}+j_{e}^{(-)}=j_{e}=\rho_{v} u\left(\frac{u^{2}}{2}+C_{p} T_{v}\right),
\end{gathered}
$$

It is easy to see that these fluxes, which completely describe this one-dimensional flow, are impossible to be characterized by any temperature. If we equate these fluxes to the corresponding thermodynamic expressions containing the velocity, temperature and density and find these values, we will see that the system of equations has two distinct complex solutions, which have no physical meaning. 
The ambiguity of the solution for real values of the thermodynamic parameters is associated with the possibility of discontinuous solutions of the type of shock wave. The complexity of the solution in this case is due to the thermodynamic non-equilibrium of the evaporation flow of Hertz-Knudsen, which can not be described in terms of thermodynamic concepts. When taking into account collisions in the non-equilibrium layer, the evaporative flux is thermalized, but the temperature on the outer side of this layer no longer coincides with the surface temperature.

\subsubsection{Approximation of the Knudsen layer}

Intense surface evaporation is essentially non-equilibrium process. In addition to the thermodynamic equilibrium, this process also has a gas-kinetic non-equilibrium in a thin (Knudsen) layer of vapor, directly adjacent to the interface. Gas-kinetic non-equilibrium is due to the flow of material through the phase boundary. The mass flow increases with the growth of the evaporation rate and, consequently, the degree of non-equilibrium of the process increases. From the physical considerations, the maximum velocity of material flow on the outside of the Knudsen layer is limited to the local speed of sound $u \leq u_{\text {sound }}=\left(\gamma R T_{v}\right)^{1 / 2}$, or $M=u / u_{\text {sound }} \leq 1$, where $u$ is the gas-dynamic velocity, $M$ is the Mach number. The maximum deviation from equilibrium is determined by the maximum value of mass flow, which is known to be achieved at $M=1$.

Under the conditions of phase equilibrium, when the saturated vapor pressure $p_{\text {sat }}$ is equal to the external pressure, the flow of vaporized material is balanced by the return flow of particles and the total mass flux through the boundary is zero. The distribution of particle velocities in vapor is in equilibrium and can be described by the Maxwell function with zero average velocity. In cases where the vapor pressure above the surface is less than the saturated vapor pressure, in the system condensed matter-vapor, the directed movement is formed with $u>0$ and is characterized by non-zero material flow through the phase boundary. The decreasing reverse flow leads to a deviation from the equilibrium in the distribution of the particles. The magnitude of the flux of returning particles decreases with the increase of the rate of evaporation, and the distribution function at the evaporation surface becomes increasingly different from Maxwellian one.

In general, the non-equilibrium distribution function is found by solving the Boltzmann equation in a region with a characteristic size of a few mean free paths. This area is adjacent to the evaporation surface, where the kinetic boundary conditions are set, taking into account the interaction of individual particles with the interface. Similar problem was solved by various methods in many studies, taking into account, in particular, the differences from unity and variability of the coefficient of condensation, which determines the probability of attachment of the particle in its collision with the evaporation surface. (See, for example. [42 - 46]).

Methods of non-equilibrium thermodynamics are used to describe the evaporation process together with other approximate phenomenological approaches [47 -52]. A more general and fundamental approach is to use molecular dynamics method, which was used in $[53,54]$ 
to analyze the evaporation process. A recent review on the issue of non-equilibrium boundary conditions at the liquid-vapor boundary is given in [55].

For the equations of continuum mechanics, thin Knudsen layer is a gas-dynamic discontinuity. The knowledge of the relations at this break, connecting the parameters of the condensed medium and the evaporated material, is needed to deal with the full gashydrodynamic problem that arises, for example, during the description of laser ablation, taking into account the variability of the Mach number and instability of the evaporation front [56]. The use of kinetic approaches, which explicitly consider the structure of the Knudsen layer, in such cases is difficult because of the emerging problem of significant difference of space-time scales. The solution of these problems is associated with the additional computational difficulties and is not always possible. Therefore, usually another approach is used that allows to determine the matching conditions with certain assumptions about the form of the non-equilibrium distribution function inside the break [57 - 60] without solving the kinetic problem. Approximation of the distribution function in the Knudsen layer was carried out in different models. But to obtain physically reasonable boundary conditions it is necessary to formulate criteria which these conditions must meet. In addition, attention is paid to the peculiarities of the behavior of the fluxes of mass, momentum and energy as the Mach number tends to unity that allows to use the requirement of extremum of total fluxes of mass $j_{m}$, momentum $j_{i}$ and energy $j_{e}$ as one of the criteria for $M=1$

" $\beta "$ - model. In " $\beta "$ - model $[61,62]$, a compound distribution function is used to describe one-dimensional non-equilibrium flow of particles on the inner side of the planar Knudsen layer: $f=f^{(+)}+f^{(-)}$. Here, the distribution $f^{(+)}$for particles, flying out of the surface is given as a Maxwell-shaped function $f^{(+)}=f\left(\rho_{\text {sat }}, T_{\text {sur }}, 0\right)$ with density of saturated vapor $\rho_{\text {sat }}$ for the surface temperature $T_{\text {sur }}$. The distribution $f^{(-)}$characterizes the flow of particles returning to the surface and is supposed to be proportional to a "shifted" Maxwell function $f^{(-)}=f\left(\rho_{v}, T_{v}, u\right)$ with density $\rho_{v}$, temperature $T_{v}$ and mean velocity $u$ steady flow of vapor on the outside of the Knudsen layer:

$$
\begin{gathered}
f^{(+)}=f\left(\rho_{\text {sat }}, T_{\text {sur }}, 0\right), \quad V_{z}>0 \\
f^{(-)}=f\left(\rho_{v}, T_{v}, u\right)=\beta\left(\frac{m}{2 \pi k_{B} T_{v}}\right)^{3 / 2} \exp \left(\frac{V_{x}^{2}+V_{y}^{2}+\left(V_{z}^{2}-u\right)^{2}}{2 k_{B} T_{v}}\right) V_{z}<0
\end{gathered}
$$

The flows of mass, momentum and energy $j_{k}=j_{k}^{(+)}+j_{k}^{(-)}, \quad k=m, i, e$ calculated using $f^{(+)}, f^{(-)}$must be equal to their gas-dynamic values $j_{k}$ that are determined by function $f$ :

$$
\begin{gathered}
\rho<V_{z}>=j_{m}^{(+)}+j_{m}^{(-)}=j_{m}=\rho_{v} u \\
\rho<V_{z}^{2}>=j_{i}^{(+)}+j_{i}^{(-)}=j_{i}=\rho_{v} R T_{v}+\rho_{v} u^{2} \\
\rho<V^{2} V_{z}>=j_{e}^{(+)}+j_{e}^{(-)}=j_{e}=\rho_{v} u\left(\frac{u^{2}}{2}+C_{p} T_{v}\right),
\end{gathered}
$$


where $C_{p}=\frac{\gamma}{\gamma+1} k_{B}$ is the vapor heat capacity per particle at constant pressure, for singleatom gas $\gamma=\frac{5}{3}$. From the solution of equations (27), it is possible to obtain gas-dynamic conditions on the break, allowing to determine the magnitude of $\rho_{v}, T_{v}$ и $\beta$ in terms of $\rho_{\text {sat }}, T_{\text {sur }}$ and $M$. The calculations showed that total fluxes $j_{m}, j_{i}, j_{e}$ have extrema depending on $M$ at $M=0.88,1.18,1.22$ correspondingly.

Thus, the requirement of the extrema of all flows in the selected point $M=1$ in the model $[61,62]$ does not met, that can serve as evidence of a bad choice of the distribution function $f^{(-)}$.

$" \varepsilon-\delta "$ - model. The drawbacks of the " $\beta "$ - model can be eliminated by selection of a more general form of the distribution function for the reverse flow of particles $f^{(-)}$[63]:

$$
f^{(-)}=\beta f+\alpha f_{1} \quad \alpha+\beta=1
$$

All 3 flows .. will have extrema at $M=1$, if the function $f_{1}$ is set to be equal to $f_{1}=f\left(\rho_{1}, T_{1}, u_{1}\right)$, where the values of $\rho_{1}, T_{1}, u_{1}$ are written in terms $\rho_{\text {sat }}, T_{\text {sur }}, \rho_{u}, T_{v}$ using additional fitting parameters $\varepsilon$ and $\delta$

$$
\begin{gathered}
\rho_{1}, T_{1}^{1 / 2}=\varepsilon \rho_{\text {sat }} T_{\text {sur }}^{1 / 2}+(1-\varepsilon) \rho_{v} T_{v}^{1 / 2}, \\
\rho_{1}, T_{1}^{1 / 2}=\varepsilon T_{\text {sur }}^{1 / 2}+(1-\varepsilon) T_{v}^{1 / 2}, \\
u_{1}=\delta M\left(\gamma R T_{1}\right)^{1 / 2}=\delta\left(T_{1} / T_{v}\right)^{1 / 2} u
\end{gathered} .
$$

For example, at $\varepsilon=0.70$ and $\delta=0.32$ all three flows $j_{k}$ will have extrema at $M=1$ with values $j_{m}=0.853, j_{i}=0.557, j_{e}=0.892$. It is clear, that this version of selection of fittings coefficients is not the only one.

" $\alpha$ " - model. It is possible to suggest another phenomenological model [63], where strict localization of extrema $j_{k}(M=1)$ is achieved without usage of fitting coefficients for such distribution function $f^{(-)}$, that do not depend on gas-dynamic values. An example of such function is function

$$
f^{(-)}=\alpha^{7} f_{0}\left(\rho_{\text {sat }}, \alpha^{2} T_{\text {sur }}\right)
$$

It takes into account the decrease of temperature $T_{\alpha}=\alpha^{2} T_{\text {sur }}$ of the reverse flow of particles as compared to the surface temperature $T_{\text {sur }}$. Due to this change of $T_{\alpha}$, the ratio of the normalized fluxes $j_{e} / j_{m}$ ceases to be a constant and takes the form $\left(1-\alpha^{8}\right)\left(1-\alpha^{10}\right)$ , which ensures that the correct limiting value is equal to 1.25 in the equilibrium case for $\alpha=1$. The equation for $\alpha$, that is obtained from the equity of fluxes (27) has a relatively simple form: 


$$
\frac{\left(1-\alpha^{8}\right)\left(1-\alpha^{10}\right)}{\left(1-\alpha^{9}\right)^{2}}=\frac{\pi}{8} \frac{\gamma^{2} M^{2}\left[(\gamma-1) M^{2}+2\right]}{(\gamma-1)\left(1+\gamma M^{2}\right)^{2}}
$$

The right-hand side of the equation (28) has a maximum at $M=1$, that determines localization of the extrema of $\alpha$ and $j_{k}$. The values of $j_{k}(M=1)$ are equal correspondingly to $j_{m}=0.85, j_{i}=0.56, j_{e}=0.90$.

Modified Crout model. The property of localization of the flows $j_{k}(M=1)$ also present in the model suggested by D. Crout [57]. It uses non-equilibrium function of distribution of particles, written in analytical form with temperature that is anisotropic by directions: $T_{L}$ and $T_{T}$.

$$
f\left(\rho, T_{L}, T_{T}, u, v, w\right)=\left\{\rho\left[\frac{\pi^{3}}{h_{T} m^{3}}\left(2 k_{B} T_{L}\right) \cdot\left(2 k_{B} T_{T}\right)\right]^{1 / 2} \cdot \exp \left[-m\left(\frac{\left(u-u_{0}\right)^{2}}{2 k_{B} T_{L}}+\frac{v^{2}}{2 k_{B} T_{T}}+\frac{w^{2}}{2 k_{B} T_{T}}\right)\right]\right\}
$$

where $T_{L}$ is the lateral temperature along $\mathrm{x}$ axis, $T_{T}$ is the transversal temperature along $\mathrm{y}, \mathrm{z}$ axis; $u, v, \omega$ are the components of the velocity vector along corresponding axes $x, y, z, \mathrm{u}_{0}$ is the drift velocity.

The modification of the Crout model [64] consists of explicit introduction of the Mach number into the main relations that allows to obtain:

$$
\begin{gathered}
T_{v}=\alpha_{\mathrm{T}}(M) T_{\text {sur }}, \quad \rho_{v}=\alpha_{\rho}(M) \rho_{\text {sat }}, \\
\alpha_{\mathrm{T}}(M)=\frac{2 \gamma M^{2}\left(m^{2}+0.5\right)^{2}}{\left(1+\gamma M^{2}\right)^{2} m^{2} t^{2}}, \quad \alpha_{\rho}(M)=\frac{1}{\exp \left(-m^{2}\right)+\pi^{1 / 2} m(1+\operatorname{erf}(m))} \cdot \frac{\left(1+\gamma M^{2}\right) m^{2}}{\gamma M^{2}\left(m^{2}+0.5\right)^{2}},
\end{gathered}
$$

The value of $m$ is determined from non-linear equation

$$
F(M)\left(m^{2}+0.5\right)^{2}-m^{2}\left(m^{2}+a+1.5\right)=0,
$$

where $F(M)=1+\frac{3 \gamma M^{2}-1}{\left(\gamma M^{2}-1\right)^{2}}, a=2 t^{2}-0.5 \pi^{1 / 2} m t-1$, .

$t=\frac{2 m}{\pi^{1 / 2}}+\frac{1+\operatorname{erf}(m)}{\exp \left(-m^{2}\right)+\pi^{1 / 2} m(1+\operatorname{erf}(m))}, \operatorname{erf}(m)=\frac{2}{\sqrt{\pi}} \int_{0}^{m} e^{-y^{2}} d y$.

For $\mathrm{M}=1: \quad T_{v}=0,633 T_{\text {sur }}, \rho_{v}=0,328 \rho_{\text {sat }} \cdot p_{v}=0.208 p_{\text {sat }}$ ，

$\mathrm{M}=0: \quad T_{v}=T_{\text {sur }}, \quad \rho_{v}=\rho_{\text {sat }}, \quad p_{v}=p_{\text {sat }}$. 
For the numerical solution of the nonlinear equation (30), one can use the Newton's iterative procedure. All fluxes $j_{m}, j_{i}, j_{e}$ have extrema at $M=1$. Calculations using an anisotropic nonequilibrium particle distribution function give the corresponding extreme values of the fluxes: $j_{m}=0.84, j_{i}=0.55, j_{e}=0.88$.

The calculations show that specific choice of the model has relatively little effect on the magnitude of the momentum flux $j_{2}$, but significantly affects the flux of mass $j_{m}$ and energy $j_{e}$. Fig.1 shows the dependencies of the ratio of the normalized fluxes $\bar{j}_{3} / \bar{j}_{1}=j_{3} j_{1}^{(+)} / j_{1} j_{3}^{(+)}$ on $M$ for all discussed models. From the comparison of the extreme values of the fluxes $j_{k}(M=1)$ and behavior of the curves $j_{m}(M), j_{e}(M)$ it follows, that due to bad choice of $f^{-1}$, the less favorable for the description of evaporation kinetics is " $\beta$ " - model.

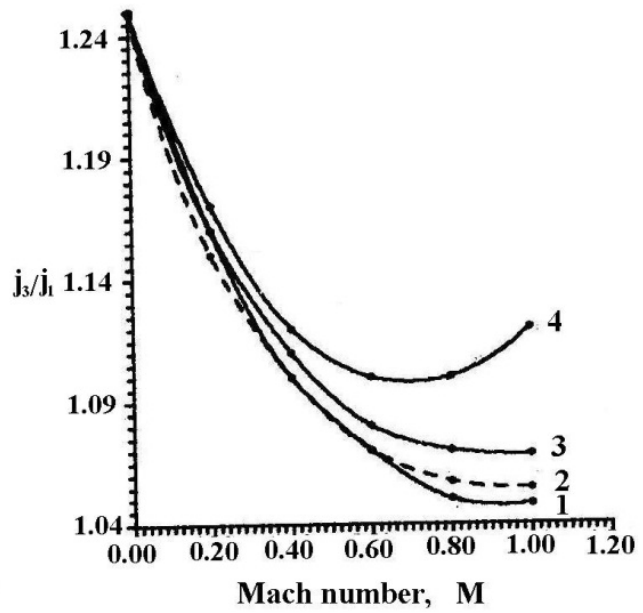

Figure 1. Dependency of the normalized ratio of energy and mass fluxes $j_{3} / j_{1}$ on Mach number for different models: 1 - " $\varepsilon-\delta "$-model, 2 - Crout model, 3 - " $\alpha$ "-model, 4 - " $\beta$ "-model (Knight).

Table 1 for all models shows numerical values of $\rho_{v}$ and $T_{v}$ (normalized by $\rho_{\text {sat }}$ and $T_{\text {sat }}$ correspondingly) depending on the Mach number that changes from zero to unity. Comparative analysis of tabular data, as well as the behavior of the curves $j_{m}, j_{e}$, show a marked difference of the values of $\rho_{v}$ and $T_{v}$, obtained using " $\beta$ " model, from the values, obtained using other models. The values of $\rho_{v}$ turned out to be underestimated, and $T_{v}$ overestimated as compared to their real values at the outer side of the Knuden layer.

\begin{tabular}{|c|c|c|c|c|c|c|c|c|}
\hline & \multicolumn{2}{|c|}{ “ $\beta$ "-model (Knight) } & \multicolumn{2}{|c|}{ “ $\varepsilon-\delta "$-model } & \multicolumn{2}{|c|}{ " $\alpha$ "-model } & \multicolumn{2}{|c|}{ Crout model } \\
\hline M & $\mathrm{T}$ & $\mathrm{N}$ & $\mathrm{T}$ & $\mathrm{N}$ & $\mathrm{T}$ & $\mathrm{n}$ & $\mathrm{T}$ & $\mathrm{n}$ \\
\hline 0.0 & 1.000 & 1.000 & 1.000 & 1.000 & 1.000 & 1.000 & 1.000 & 1.000 \\
\hline 0.1 & 0.960 & 0.861 & 0.958 & 0.864 & 0.960 & 0.869 & 0.953 & 0.861 \\
\hline 0.2 & 0.922 & 0.748 & 0.916 & 0.753 & 0.920 & 0.758 & 0.910 & 0.749 \\
\hline 0.3 & 0.866 & 0.654 & 0.876 & 0.662 & 0.881 & 0.666 & 0.870 & 0.658 \\
\hline
\end{tabular}




\begin{tabular}{|c|cc|cc|cc|cc|}
\hline & \multicolumn{2}{|c|}{ “ $\beta$ "-model (Knight) } & \multicolumn{2}{|c|}{ “ $\varepsilon$ - $\delta$ ”-model } & \multicolumn{2}{c|}{ “ $\alpha$ "-model } & \multicolumn{2}{c|}{ Crout model } \\
\hline $\mathrm{M}$ & $\mathrm{T}$ & $\mathrm{N}$ & $\mathrm{T}$ & $\mathrm{N}$ & $\mathrm{T}$ & $\mathrm{n}$ & $\mathrm{T}$ & $\mathrm{n}$ \\
\hline 0.4 & 0.851 & 0.576 & 0.837 & 0.587 & 0.844 & 0.588 & 0.833 & 0.582 \\
\hline 0.5 & 0.817 & 0.511 & 0.799 & 0.525 & 0.808 & 0.524 & 0.798 & 0.519 \\
\hline 0.6 & 0.785 & 0.457 & 0.763 & 0.472 & 0.773 & 0.470 & 0.763 & 0.466 \\
\hline 0.7 & 0.754 & 0.410 & 0.727 & 0.428 & 0.740 & 0.424 & 0.730 & 0.421 \\
\hline 0.8 & 0.724 & 0.371 & 0.693 & 0.391 & 0.705 & 0.386 & 0.697 & 0.384 \\
\hline 0.9 & 0.696 & 0.337 & 0.660 & 0.360 & 0.672 & 0.355 & 0.665 & 0.352 \\
\hline 1.0 & 0.669 & 0.308 & 0.628 & 0.333 & 0.640 & 0.328 & 0.633 & 0.326 \\
\hline
\end{tabular}

Table 1. Normalized values $\rho_{v}$ and $T_{v}$ depending on the Mach number for different models

The modified Crout model, " $\varepsilon-\delta "-$ model, " $\alpha "$ - model fulfill the requirement of the extremum of the flows $j_{k}$ at $M=1$. The difference of the results that were obtained using these models does not exceed $1.5 \%$. Any of these models can be used to describe the kinetics of the process of non-equilibrium surface evaporation.

\subsection{Conclusions}

The performed brief analysis of the kinetics of phase transitions is an introduction to the construction of the models that combine mathematical description of kinetics of high-speed phase transformations with dynamics of the macro-processes (heat and mass transfer) under conditions of a strong deviation from local thermodynamic equilibrium that are typical for ultra-short super-power laser action on metals.

\section{Thermodynamic and thermo-physical properties of phonon and electron Fermi gas}

Determination of physical characteristics of a medium, including equations of state under conditions of local thermodynamic equilibrium can be carried out either experimentally or by means of calculation using distribution functions - a Maxwell-Boltzmann function for ideal gas and ideal plasma and Fermi one for degenerate electron gas, and for the phonongas - Bose function. In case of violation of the conditions of local thermodynamic equilibrium distribution functions are determined by solving the classical kinetic Boltzmann equation or quantum-kinetic equations. The presence of distribution function is just the required minimum of information that can be used to describe nonequilibrium processes with reasonable accuracy.

The influence of ultrashort high-energy laser on a strongly absorbing media (metals, semiconductors) is in a very short temporal and spatial scales and leads to disturbance of their general local-thermodynamic equilibrium. Irradiated targets in these conditions are presented in the form of two subsystems - electron and phonon each of which is in local thermodynamic equilibrium and are characterized by their temperatures and equations of state. As a 
consequence, all processes are described in the two-temperature approximation [65], [66]. The target at pico- and femtosecond influence may be heated to very high temperatures and pressures at which the thermal and mechanical properties of matter are not known in general. One of the most important problems for the mathematical modeling is the necessity to determine thermophysical, optical and thermodynamic properties in a wide (tens and hundreds of electronvolts) temperature and frequency ranges for each of the subsystems.

\subsection{Electron subsystem}

The most important thermophysical and thermodynamic characteristics of the electron Fermi gas within the scope of heat-conducting mechanism of energy transfer are: heat capacity $C_{e}$, thermal diffusivity $\chi_{e}$ and thermal conductivity $\lambda_{e}$. For its determination using fundamental physical quantities, which include the electron mean free paths $l_{e e}, l_{e p h}$ and the characteristic times (frequency) of interaction for two scattering mechanisms: the electron-electron $\tau_{e e}$ and electron-phonon $\tau_{e p h}$.

\subsubsection{Fermi-Dirac integral and its approximation}

For a quantitative description of electrical, thermodynamic and thermophysical properties of degenerate electron gas with distribution function

$$
f_{e}=\frac{1}{\exp \left(\frac{\varepsilon_{e}-\mu}{k_{B} T_{e}}\right)+1}
$$

widely used Fermi-Dirac functions $F_{k+1 / 2}\left(\mu\left(T_{e}\right) / T_{e}\right)$ expressed in terms of integrals of the form

$$
F_{k+1 / 2}\left(\mu\left(T_{e}\right) / T_{e}\right)=\int_{0}^{\infty} \frac{\left(\frac{\varepsilon_{e}}{k_{B} T_{e}}\right)^{k+1 / 2} d\left(\frac{\varepsilon_{e}}{k_{B} T_{e}}\right)}{\exp \left(\frac{\varepsilon_{e}-\mu}{k_{B} T_{e}}\right)+1}=\int_{0}^{\infty} \frac{E^{k+1 / 2} d E}{\exp \left(E-\frac{\mu}{k_{B} T_{e}}\right)+1},
$$

where $T_{e}, \varepsilon_{e}$ - temperature and energy of electron, $\mu\left(T_{e}\right)$ - chemical potential, $\mu(0)=\varepsilon_{F}-$ Fermi energy.

In the future, the Fermi integrals will be represented as a function of dimensionless energy of the chemical potential:

$$
F_{k+1 / 2}(\eta)=\int_{0}^{\infty} \frac{E^{k+1 / 2} d E}{\exp (E-\eta)+1},
$$

where $E=\varepsilon_{e} / k_{B} T_{e}, \eta\left(T_{e}\right)=\mu\left(T_{e}\right) / k_{B} T_{e}$ - dimensionless energy and chemical potential of electrons. 
The integral of form $F_{1 / 2}(\eta)=\int_{0}^{\infty} \frac{E^{1 / 2} d E}{\exp (E-\eta)+1}$ is used to determine the electron density $N_{e}=\int_{0}^{\infty} g(E) f(E) d E=\int_{0}^{\infty} \frac{g(E) d E}{\exp (E-\eta)+1}=\int_{0}^{\infty} F(E) d E$,

where $\quad g(E)=\frac{2^{1 / 2} m^{3 / 2}}{\pi^{2} \hbar^{3}}\left(k_{B} T_{e}\right)^{3 / 2} E^{1 / 2}=g_{0} \cdot\left(k_{B} T_{e}\right)^{3 / 2} E^{1 / 2} \quad$ is $\quad$ a density of states, $F(E)=g(E) f(E)=g_{0}\left(k_{B} T_{e}\right)^{3 / 2} \frac{E^{1 / 2}}{\exp (E-\eta)+1}$ is distribution function of the free electron energy, $g_{0}=\frac{2^{1 / 2} m^{3 / 2}}{\pi^{2} \hbar^{3}}$. Then

$$
N_{e}=g_{0} \cdot\left(k_{B} T_{e}\right)^{3 / 2} \int_{0}^{\infty} \frac{E^{1 / 2} d E}{\exp (E-\eta)+1}=g_{0} \frac{2}{3} \varepsilon_{F}^{3 / 2}=g_{0} \cdot\left(k_{B} T_{e}\right)^{3 / 2} F_{1 / 2}(\eta)
$$

From the known distribution function of particle energy $F(E)$, using the ratio $<x(E)>=\frac{\int_{0}^{\infty} x(E) F(E) d E}{\int_{0}^{\infty} F(E) d E}$ the average value of any physical quantity depends on energy can be found. Since the average energy of the electron gas is defined as the ratio of Fermi integrals

$$
<\varepsilon_{e}>=\frac{\int_{0}^{\infty} E \cdot F(E) d E}{\int_{0}^{\infty} F(E) d E}=\left(k_{B} T_{e}\right) \frac{\int_{0}^{\infty} \frac{E^{3 / 2} d E}{\exp (E-\eta)+1}}{\int_{0}^{\infty} \frac{E^{1 / 2} d E}{\exp (E-\eta)+1}}=\left(k_{B} T_{e}\right) \frac{F_{3 / 2}(\eta)}{F_{1 / 2}(\eta)} .
$$

Similarly, we can determine the other physical quantities of the electron gas.

The chemical potential $\mu\left(T_{e}\right)$ depends on the temperature, so the Fermi integrals can be conveniently represented as a function of dimensionless temperature $\xi=\frac{k_{B} T_{e}}{\varepsilon_{F}}$. In [67] for the integrals of the form (33) has been proposed convenient approximation, which allows to express the integrals $F_{k+1 / 2}(\xi)$ through the transcendental gamma-functions $\Gamma(k+1 / 2)$ and the dimensionless temperature $\xi$ :

$$
F_{k+1 / 2}(\xi)=A \xi^{-3 / 2}\left[1+(B / \xi)^{2}\right]^{k / 2}
$$


where $A=\frac{2}{3} \frac{\Gamma\left(k+\frac{3}{2}\right)}{\Gamma\left(\frac{3}{2}\right)}, \quad B=\left[A\left(k+\frac{3}{2}\right)\right]^{-1 / k}$ coefficients expressed in terms of gammafunctions. Equation (36) has correct asymptotics at $\xi \rightarrow 0$ and $\xi \rightarrow \infty$ :

$$
\begin{array}{ll}
F_{k+1 / 2}(\eta) \approx 1 / \xi^{k+3 / 2}(k+3 / 2), & \xi \rightarrow 0 \\
F_{k+1 / 2}(\eta) \approx \frac{2}{3} \frac{\Gamma(k+3 / 2)}{\Gamma(3 / 2)} \frac{1}{\xi^{3 / 2}} & \xi \rightarrow \infty
\end{array}
$$

The integral of $k=1 / 2$ order can easily be determined from the expression (34): $F_{1 / 2}(\xi)=\frac{2}{3} \frac{1}{\xi^{3 / 2}}$.

The most frequently used Fermi integrals that are expressed through (36) have the form:

$$
F_{-1 / 2}(\xi)=\frac{4}{3} \frac{\xi^{-1 / 2}}{\left(\xi^{2}+\frac{4}{9}\right)^{1 / 2}}, F_{3 / 2}(\xi)=\frac{\left(\xi^{2}+\left(\frac{2}{5}\right)^{2}\right)^{1 / 2}}{\xi^{5 / 2}}, F_{5 / 2}(\xi)=\left[\frac{5}{2} \xi^{-3 / 2}+\frac{2}{7} \xi^{-7 / 2}\right] .
$$

The maximum error compared with the exact solution [68] for integrals at $k=-1$ and $k=1$ does not exceed $8 \%$, but increases slightly with increasing of $k$.

Approximation (36) allows to obtain simple analytical expressions for the physical quantities of the electron gas at arbitrary temperatures.

\subsubsection{Equations of state}

Using the approximating expressions (36) and (37) equations of state for degenerate electron gas can be written as simple analytical expressions at arbitrary temperatures. Since the average electron energy $\left\langle\varepsilon_{e}\right\rangle$ and its pressure can be represented as

$$
\begin{gathered}
<\varepsilon_{e}>=T_{e} \frac{F_{3 / 2}}{F_{1 / 2}}=\frac{3}{2} \varepsilon_{F}\left(\xi^{2}+0.16\right)^{1 / 2}, \\
p=\frac{2}{3} N_{e}<\varepsilon_{e}>=\frac{2}{3} N_{e} T_{e} \frac{F_{3 / 2}}{F_{1 / 2}}=N_{e} \varepsilon_{F}\left(\xi^{2}+0.16\right)^{1 / 2},
\end{gathered}
$$

\subsubsection{Electron heat capacity $C_{e}\left(T_{e}\right)$}

The expression for the heat capacity of the electron gas $C_{e}\left(T_{e}\right)=\frac{\partial}{\partial T}\left(N_{e}\left\langle\varepsilon_{e}\right\rangle\right)$ can be obtained from the relations 


$$
\frac{\partial}{\partial \eta} F_{k+1 / 2}=(k+1 / 2) F_{k-1 / 2}, \frac{\partial \eta}{\partial T}=\frac{3}{T} \frac{F_{1 / 2}}{F_{-1 / 2}}, C_{e}\left(T_{e}\right)=\frac{N_{e}}{F_{1 / 2}}\left(\frac{5}{2} F_{3 / 2}-\frac{9}{2} F_{-1 / 2}\right) .
$$

Using the approximating expressions (37), heat capacity of electron gas can be represented with an error not exceeding $5 \%$ as the following function

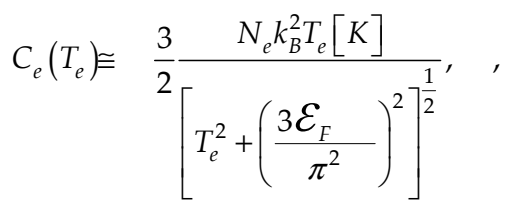

where $N_{e}=z N_{a}, z$ - the number of valence electrons, $N_{a}$ - the concentration of atoms (ions) of lattice.

The resulting expression gives the classical linear dependence of heat capacity of a degenerate electron gas vs. temperature [69] in low temperature region $T_{e}<<\varepsilon_{F}$, $C_{e}=\frac{\pi^{2}}{2} \frac{k_{B}^{2} T_{e} N_{e}}{\varepsilon_{F}}$ and constant value at $T_{e} \gg \varepsilon_{F}$ equal to heat capacity of gas with Maxwell distribution $C_{e}=3 / 2 k_{B} N_{e}$. Dependences for copper and aluminum are shown in Fig. 2.

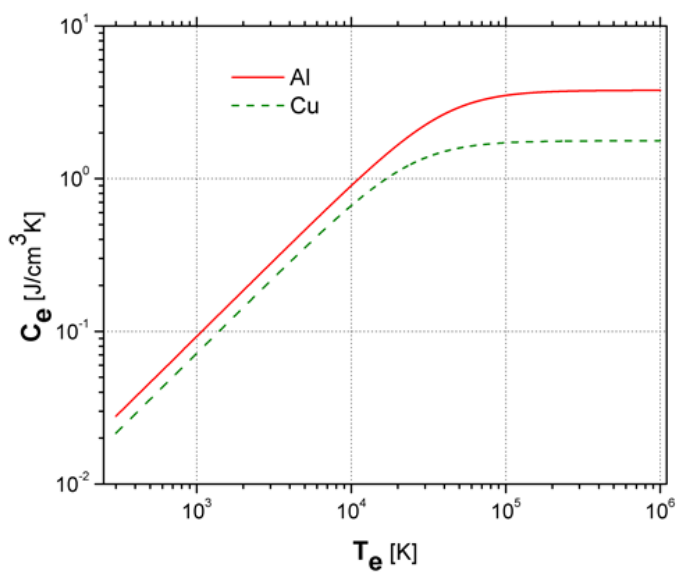

Figure 2. Temperature dependence of electron heat capacity $C_{e}\left(T_{e}\right)$.

\subsubsection{The thermal diffusivity of a degenerate electron gas $\chi_{e}$.}

The thermal diffusivity of electron gas is proportional to the product of the mean free path $l_{e}$ and average velocity of the electron $\left\langle v_{e}\right\rangle$ :

$$
\chi_{e}=\frac{1}{3} l_{e}<v_{e}>
$$


In metals electron mean free path due to several mechanisms: pair of electron-electron collisions, electron-phonon collisions and scattering by plasmons.

Electron-electron collisions dominate at temperatures comparable to the Fermi energy $T_{e} / \varepsilon_{F} \approx 1$. Electron-phonon interaction is dominant at low temperatures $T_{e} / \varepsilon_{F} \ll 1$. The interaction associated with the excitation of plasmons occurs at high temperatures, exceeding the plasma frequency energy $T_{e}>\hbar \omega_{r}\left(\hbar \omega_{r} \cong 10-20 \mathrm{eV}\right)$. Taking into account high temperature region of occurrence and limitation of experimental data about reducing of the mean free path of electrons due to plasmon excitation (it is known only for some metals), electron scattering by plasmons will not be considered.

\subsubsection{The electron-electron thermal diffusivity $\chi_{e e}\left(T_{e}\right)$}

The mean free path of an electron in pair electron-electron collisions $l_{e e}$ is determined from the known gas-dynamic formula

$$
l_{e e}=\frac{1}{N_{e} \sigma_{e e}},
$$

where $\sigma_{e e}$ - scattering cross section with energy $\Delta \mathcal{E}$ transfer for electrons with energies $\varepsilon_{1}, \varepsilon_{2}$. The cross section $\sigma_{e e}$ is expressed through the transport cross section of the collision of two isolated electrons $\sigma_{\ell \ell}^{t r}$ and Fermi integrals $F_{-1 / 2}, F_{1 / 2}$. In turn, the transport cross section of collision of two isolated electrons $\sigma_{\ell \ell}^{t r}$ in a field of screened Coulomb potential $U=\frac{e^{2}}{r} \exp \left(-\frac{r}{d}\right)$ is expressed through the differential scattering cross section $d \sigma$ determined in the Born approximation [70, p.560]. The final cross sections will be written in form

$$
\begin{gathered}
\sigma_{e e}^{t r}=\frac{2 \pi}{9}\left(\frac{4}{9 \pi}\right)^{4 / 3} z^{-4 / 3} \frac{r^{4}}{r_{B}^{2}} \frac{\left[\ln (t+1)-\frac{t}{(t+1)}\right]}{\left(\xi^{2}+0.16\right)}, \\
\sigma_{e e}=\sigma_{e e}^{t r}\left(\frac{F_{-1 / 2}}{2 F_{1 / 2}}\right)^{2}=\frac{2 \pi}{9}\left(\frac{4}{9 \pi}\right)^{4 / 3} z^{-4 / 3} \frac{r^{4}}{r_{B}^{2}} \frac{\xi^{2}\left[\ln (t+1)-\frac{t}{(t+1)}\right]^{\prime}}{\left(\xi^{2}+\frac{4}{9}\right)\left(\xi^{2}+0.16\right)}
\end{gathered}
$$

where $t=4\langle k\rangle^{2} d^{2}=\left(\frac{9 \pi}{4}\right)^{4 / 3} z^{1 / 3} \frac{r_{B}}{r} \cdot\left[\left(\xi^{2}+0.16\right) \cdot\left(\xi^{2}+\frac{4}{9}\right)\right]^{1 / 2}, r_{B}=\frac{\hbar^{2}}{m e^{2}}=0.52910^{-8} \mathrm{~cm}-\mathrm{Bohr}$ radius, $r=\left(\frac{3}{4 \pi N_{a}}\right)^{1 / 3}$ - the average distance between atoms, $e$ - the electron charge, $d$ - field acting radius (Debye) $U=\frac{e^{2}}{r} \exp \left(-\frac{r}{d}\right)$. 
At low temperatures $T_{e}<<\varepsilon_{F}$, the effective cross section $\sigma_{e e}$ is small and amounts to $\sigma_{e e}=\sigma_{e e}^{t r} \frac{\xi^{2}}{\left(\xi^{2}+4 / 9\right)} \approx \sigma_{e e}^{t r} \cdot\left(\frac{T_{e}}{\varepsilon_{F}}\right)^{2}$. Maximum of cross section is achieved at $T_{e} \geq \varepsilon_{F}$, Fig.3, when the degeneracy is passed and the electron-electron collisions with large energy transfer become possible. At very high temperatures $T_{e} \gg \mathcal{E}_{F}$ cross section becomes the Coulomb one, Fig.3, and decreases logarithmically. The mean free path of electrons $l_{e e}$ is determined by the formula (42):

$$
\begin{aligned}
l_{e e} & =\frac{1}{N_{e} \sigma_{e e}}=\left[N_{e} \cdot \frac{2 \pi}{9}\left(\frac{4}{9 \pi}\right)^{4 / 3} z^{-4 / 3} \frac{r^{4}}{r_{B}^{2}} \frac{\xi^{2}\left[\ln (t+1)-\frac{t}{(t+1)}\right]}{\left(\xi^{2}+\frac{4}{9}\right)\left(\xi^{2}+0.16\right)}\right]^{-1}= \\
& =\frac{2}{\pi^{2}}\left(\frac{4}{9 \pi}\right)^{1 / 3} \frac{1}{r^{2}} \frac{1}{z^{1 / 3} N_{a}} \cdot \frac{t^{2}}{\xi^{2}}\left[\ln (1+t)-\frac{t}{1+t}\right]^{-1}
\end{aligned}
$$

Calculations indicate that the mean free path for $A l$ and $C u$ change in a wide range $\left(\sim 10^{-2} \div\right.$ $\left.10^{-7}\right) \mathrm{cm}$ and have a minimum at $T_{e} \approx \varepsilon_{F}$.

The average thermal velocity of electron $\left\langle v_{e}\right\rangle$ is expressed through its average energy $<\varepsilon_{e}>$ :

$$
<v_{e}>=\left(\frac{2}{m}<\varepsilon_{e}>\right)^{1 / 2}=\left(\frac{3 \varepsilon_{F}}{m}\left(\xi^{2}+0.16\right)^{1 / 2}\right)^{1 / 2}=\left(\frac{3}{2}\right)^{1 / 2}\left(\frac{9 \pi}{4}\right)^{1 / 3} \frac{z^{1 / 3}}{r} \frac{\hbar}{m_{e}}\left(\xi^{2}+0.16\right)^{1 / 4}
$$

Taking into account (44) and (45) electronic thermal diffusivity $\chi_{e e}$ takes the form:

$$
\begin{aligned}
& \chi_{e e}=\frac{1}{3} l_{e e}<v_{e}>=\frac{1}{3} \frac{2}{\pi^{2}}\left(\frac{4}{9 \pi}\right)^{1 / 3} \frac{1}{r^{2}} \frac{1}{z^{1 / 3} N_{a}} \cdot \frac{t^{2}}{\xi^{2}}\left[\ln (1+t)-\frac{t}{1+t}\right]^{-1} \cdot\left(\frac{3}{2}\right)^{1 / 2}\left(\frac{9 \pi}{4}\right)^{1 / 3} \\
& \frac{z^{1 / 3}}{r} \frac{\hbar}{m_{e}}\left(\xi^{2}+0.16\right)^{1 / 4}=\left(\frac{2}{3}\right)^{1 / 2} \frac{4}{3 \pi} \frac{\hbar}{m_{e}} \frac{t^{2}}{\xi^{2}} \frac{\left(\xi^{2}+0.16\right)^{1 / 4}}{\left[\ln (1+t)-\frac{t}{1+t}\right]}=\chi_{0} \cdot \Phi_{e e}(\xi)
\end{aligned}
$$

where $\Phi_{e e}(\xi)=\frac{t^{2}}{\xi^{2}} \frac{\left(\xi^{2}+0.16\right)^{1 / 4}}{\left[\ln (1+t)-\frac{t}{1+t}\right]}$ is dimensionless function,

$\chi_{0}=\sqrt{\frac{2}{3}} \frac{4}{3 \pi} \frac{\hbar}{m_{e}}=0.402,\left[\frac{\mathrm{cm}^{2}}{\mathrm{~s}}\right]$. 
Fig. 4 shows the temperature dependence of $\chi_{e e}\left(T_{e}\right)$ for $A l$ and $C u$. Temperature dependence of electron-electron thermal diffusivity $\chi_{e e}\left(T_{e}\right)$ of both metals has a deep minimum at $T_{e} \approx \varepsilon_{F}$. Its value reaches $\sim 20 \div 30\left[\mathrm{~cm}^{2} / \mathrm{s}\right]$, Fig.4. At removal of degeneracy, when $T_{e}>\varepsilon_{F}$ the thermal diffusivity increases due to decreasing of the effective cross section $\sigma_{e e}$. With further increase of temperature $T_{e} \gg \varepsilon_{F}$ the thermal diffusivity continues to increase and its dependence $\chi_{e e} \sim \xi^{5 / 2} / \ln \xi^{2}$ coincides with temperature dependence of thermal diffusivity of Maxwell electron plasma. At low temperature $T_{e} \ll \varepsilon_{F}$ dependence of thermal diffusivity is inversely proportional to square of temperature $\chi_{e e} \sim T_{e}^{-2}$. Strong growth of $\chi_{e e}\left(T_{e}\right)$ with decreasing of $T_{e}$ leads to the fact that $\chi_{e e}$ reach $(1-5) \cdot 10^{5} \frac{\mathrm{cm}^{2}}{\mathrm{~s}}$ values which is 3-4 orders higher than the actual electron thermal diffusivity of metals at room temperatures. Thus, the resulting expression (46) is a good approximation only for high temperatures. Under normal conditions $\left(T_{e} \sim 300 \mathrm{~K}\right)$ it is necessary to consider the scattering of electrons of metals by phonons to determine thermal diffusivity, this interaction is dominant at low temperatures.

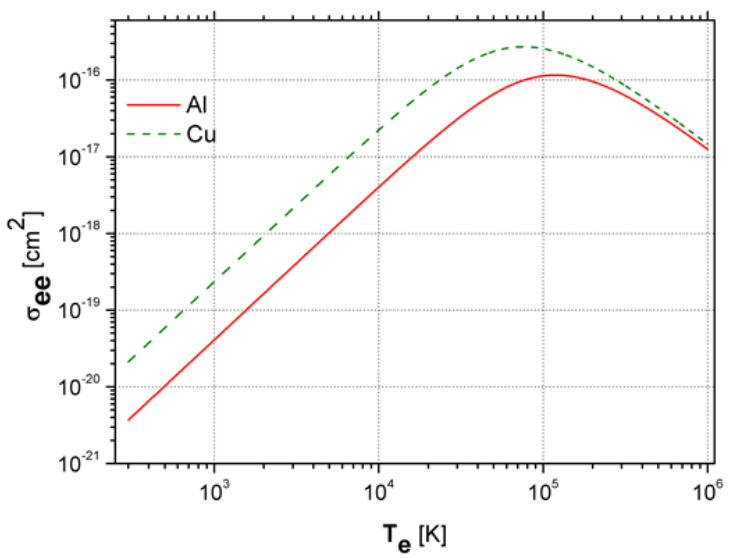

Figure 3. Temperature dependences of electron-electron scattering cross section $\sigma_{e e}\left(T_{e}\right)$.

\subsubsection{The electron-phonon thermal diffusivity}

The mean free path, defined by electron-phonon interaction is described by the assumption of elastic scattering of conduction electrons of metal on lattice oscillations. To determine it is convenient to use the phenomenological approach [70], in which the crystal is considered as an elastic continuum. Lattice oscillations at the same time considered as a wave of elastic deformations. To simplify the density fluctuations are presented as deviations of each atom (ion) from the average, which square of amplitude is directly proportional to temperature $T_{p h}$. According to the macroscopic theory of 
elasticity we can obtain an expression for the mean free path, expressed in terms of macroscopic quantities [71] by expressing the force tending to return the atom (ion) to the equilibrium state through the Young's modulus E:

$$
\ell_{e p h}=\frac{E \cdot r}{N_{e} k_{B} T_{p h}}
$$

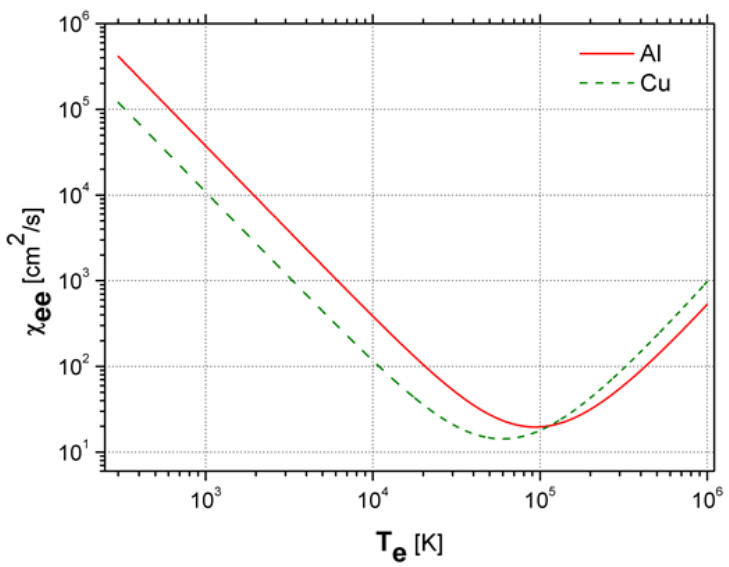

Figure 4. Temperature dependence of electron-electron thermal diffusivity $\chi_{e e}\left(T_{e}\right)$.

From the expression (47) it follows that $\ell_{e p h}$ is inversely proportional to the lattice temperature $T_{p h}$ in electron-phonon interaction.

During melting of a metal the number of collectivized electrons remains practically unchanged. The modulus of elasticity is only one value (excluding the jump in specific volume, which usually does not exceed $10 \%$ ) that changes. The melting of most metals is accompanied by decrease in elastic modulus by 2-3 times [72]. This decrease causes a corresponding increase in density fluctuation and, consequently, an abrupt decrease in the mean free path $\ell_{e p h}$ :

$$
\left(\ell_{e p h}\right)_{k}=\left(\frac{E \cdot r}{z N_{a} k_{B} T_{p h}}\right)_{k}
$$

where $k=s, l$ subscripts denoting membership in the solid and liquid phases, respectively, $r=\left(\frac{3}{4 \pi} \frac{1}{N_{a}}\right)^{1 / 3}=0.6204 N_{a}^{-1 / 3}$ is a distance between the atoms. From below $l_{e p h}$ is limited by Bohr diameter value. The mean free path of electrons taking into account the scattering by phonons, calculated for aluminum and copper from the relation (48) showed that, compared with the mean free path of the electron-electron scattering values for both metals 
decreased by several orders of magnitude: for the high-temperature region of $1.5 \div 2$ orders of magnitude, while at low temperatures for $4 \div 5$ orders of magnitude.

The thermal diffusivity $\chi_{e p h}\left(T_{e}, T_{p h}\right)$, defined by the mechanism in electron-phonon interaction is calculated by the formula:

$$
\left(\chi_{e p h}\left(T_{e}, T_{p h}\right)\right)_{k}=\left(\frac{1}{3} \ell_{e p h}\left(T_{p h}\right)<v_{e}\left(T_{e}\right)>\right)_{k},
$$

Dependences $\chi_{e p h}\left(T_{e}, T_{p}\right)$ for $A l$ and $C u$ calculated from (49) at $T_{e}=T_{p h}$ are shown in Fig.5. The calculations show that at room temperature values of the electron thermal diffusivity $\chi_{e p h}$, for both materials are 3-4 orders of magnitude less than the thermal diffusivity $\chi_{e e}$ and reach values of $10^{2} \mathrm{~cm}^{2} / \mathrm{s}$ typical for metals under normal conditions. Under equilibrium conditions with temperature $T_{p h}$ increasing, thermal diffusivity $\chi_{\text {eph }}$, undergoing break at the phase transition decreases rapidly to a value of $\sim 1 \mathrm{~cm}^{2} / \mathrm{s}$ at a temperature of $\sim 5000 \mathrm{~K}$. Function $\chi_{e p h}\left(T_{e}, T_{p}\right)$ increase by several orders of magnitude with electron temperature increase.

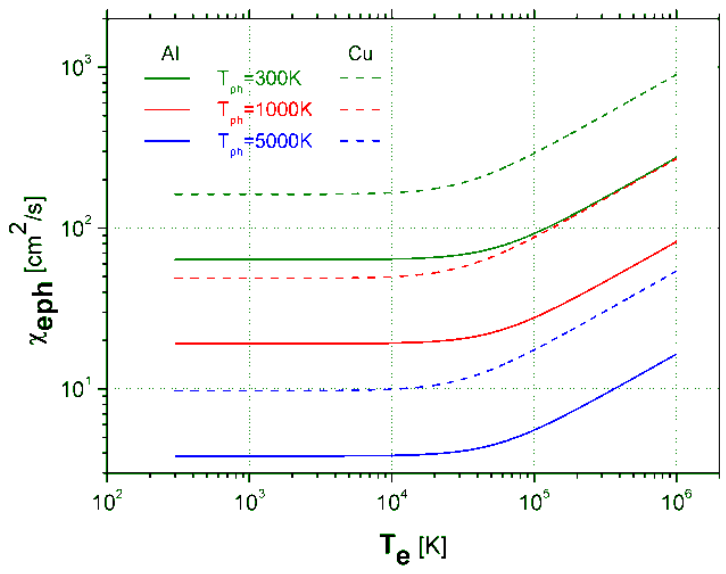

Figure 5. Temperature dependences of thermal diffusivity $\chi_{e p h}\left(T_{e}, T_{p h}\right)$ for $A l$ and Cu with fixed $T=T_{e}=T_{p h}$.

\subsubsection{The resulting thermal diffusivity of electron Fermi gas $\chi_{e}\left(T_{e}, T_{p h}\right)$}

Calculations have shown that taking into account only a pair electron-electron collisions leads to a strong (by several orders of magnitude) overestimation of the thermal diffusivity of the electron gas at low temperatures $T_{e} \ll \varepsilon_{F}$. Accounting for electron-phonon collisions gives a more realistic values of $\chi_{e}\left(T_{e}\right)$ at low temperatures.

By averaging mean free paths $\ell_{e e}, \ell_{e p h}$ we obtain an expression for resulting thermal diffusivity, at arbitrary temperature 


$$
\left(\chi_{e}\left(T_{e}, T_{p h}\right)\right)_{k}=\left(\frac{\chi_{e e} \chi_{e p h}}{\chi_{e e}+\chi_{e p h}}\right)_{k}=\left(\frac{1}{3} \ell_{e}<v_{e}>\right)_{k}=<v_{e}>\left(\frac{\ell_{e e} \ell_{e p h}}{3\left(\ell_{e e}+\ell_{e p h}\right)}\right)_{k=s, \ell},
$$

Fig.6 shows temperature dependences of total electron thermal diffusivity $\chi_{e}\left(T_{e}, T_{p h}\right)$ for $A l$ and $C u$ in equilibrium $T_{e}=T_{p}$.

\subsubsection{The thermal conductivity of the electron gas}

According to elementary kinetic theory, the thermal conductivity of the gas is

$$
\left(\lambda_{e}\left(T_{e}, T_{p h}\right)\right)_{k}=\left(\frac{1}{3} C_{e} \ell_{e}\left(T_{e}, T_{p h}\right)<v_{e}>\right)_{k}=\left(C_{e} \chi_{e}\left(T_{e}, T_{p h}\right)\right)_{k},\left[\frac{\mathrm{W}}{m \cdot K}\right]
$$

Thus, the thermal conductivity $\lambda_{e}$ can be determined through the heat capacity $C_{e}$ and averaged thermal diffusivity of electron gas $\chi_{e}\left(T_{e}, T_{p h}\right)$. The temperature dependences of $\lambda_{e}$ for $A l$ and $C u$ calculated for the equilibrium case when $T_{e}=T_{p h}$ are shown in Fig.6. In accordance with the results, the equilibrium electron thermal conductivity $\lambda_{e}\left(T_{e}, T_{p h}\right)$ at temperatures not exceeding the boiling temperature of the equilibrium is practically independent of temperature. In high temperature region $T_{e}>1 \mathrm{eV}$ thermal conductivity increases rapidly due to the dominance of electron-electron scattering. It is natural that in this region the thermal conductivity of the electron gas depends on electron density and $\lambda_{e}$ increases with increasing of electron concentration. For this reason, electron thermal conductivity of aluminum is higher than the same one of copper at the high-temperature. In low temperature region where electron-phonon interaction is dominated, the ratio is inverse. Because of the greater mean free path $\ell_{e p h}$, electron thermal conductivity of copper is higher than that of aluminum.

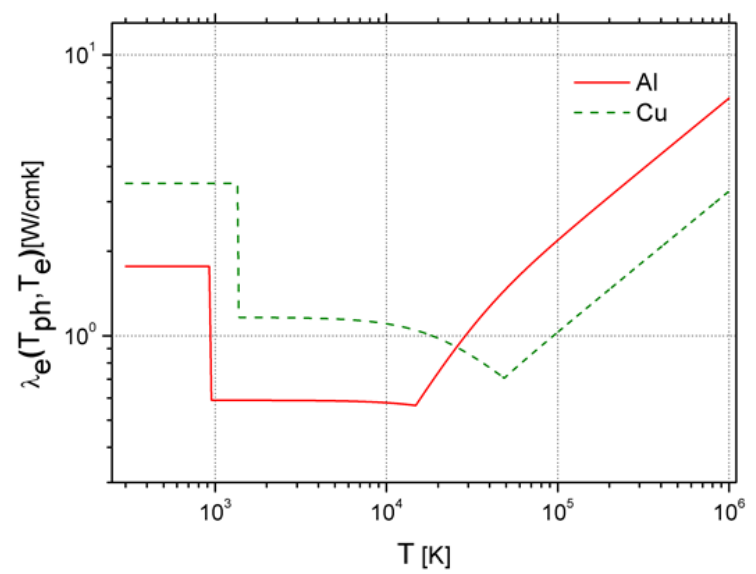

Figure 6. Temperature dependence of total electron thermal conductivity $\lambda_{e}\left(T_{e}, T_{p h}\right)$. 


\subsection{Phonon gas}

\subsubsection{Heat capacity of phonons}

The main consequence of the existence of lattice oscillations is the possibility of its thermal excitation, which is appeared as a contribution to the heat capacity of solid.

Taking into account the process of melting, heat capacity of phonon gas can be written as [69], [73]:

$$
C_{p h, k}= \begin{cases}3 k_{B} N_{a, k^{\prime}} \quad k=s, l, & T>T_{D} \\ \frac{12 \pi^{4}}{5} N_{a} k_{B}\left(\frac{T_{p h}}{T_{D}}\right)^{3}, & T<T_{D}{ }^{\prime},\left[\frac{J}{m^{3} K}\right]\end{cases}
$$

$T_{D}$ is a Debye temperature.

\subsubsection{The thermal diffusivity of phonon gas $\chi_{p h}$}

Phonons are considered as a gas of particles. From elementary kinetic theory, thermal diffusivity of a gas is given by

$$
\chi_{\text {ph }}=\frac{1}{3} \ell_{\text {ph }} v_{\text {sound }}
$$

where $\ell_{p h}$ - phonon mean free path. It is assumed that the phonons move with velocity of sound $v_{\text {sound }}$.

The mean free path of phonons $\ell_{p h}$ is determined from the description of thermal motion in a solid by means of notions of the phonon gas. The interaction between the phonons can be characterized by some effective cross section which is proportional to the mean square of thermal expansion of the body or the mean square of density fluctuation $\rho$ [69]: $\Delta^{2}=N_{a} k_{B} T_{p} \beta$, where $\beta$ is a coefficient of compressibility. Taking into account $\beta=\left(\rho v_{\text {sound }}^{2}\right)^{-1}$ :

$$
\Delta^{2}=N_{a} k_{B} T_{p h} \beta=\frac{N_{a} k_{B} T_{p h}}{\rho v_{\text {sound }}^{2}}=\frac{k_{B} T_{p h}}{M v_{\text {sound }}^{2}}
$$

Assigning to phonons radius equal to thermal oscillations amplitude, we can taken into account scattering of sound waves and determine the mean free path $\ell_{p h}$

$$
\ell_{p h}=\frac{r}{\Delta^{2} \gamma^{2}}=\frac{M v_{s}^{2}}{k_{B} T_{p h}} \frac{r}{\gamma^{2}}
$$


where $M$ - the mass of the atom, $\gamma$ - Griuneyzen constant.

Accounting for the expressions for the mean free path $\ell_{p h}$ (54) and the velocity of sound $v_{\text {sound }}[73]$ expressed through the Fermi velocity

$$
v_{\text {sound }}=\left(\frac{m}{3 M}\right)^{1 / 2} v_{F}=\left(\frac{m}{3 M}\right)^{1 / 2}\left(\frac{9 \pi}{4} z\right)^{1 / 3} \frac{\hbar}{m} \frac{1}{r}, M=M_{0} A ，
$$

thermal diffusivity of phonons can be written as:

$$
\chi_{p h}=\frac{1}{3} \ell_{p h} v_{s}=\frac{M_{0} A v_{S}^{3}}{3 k_{B} T_{p h}} \frac{r}{\gamma^{2}}=\left(\frac{3}{4 \pi}\right)^{1 / 3} \frac{\pi^{2}}{(3 m)^{3 / 2}} \frac{\hbar^{3}}{\left(M_{0} A\right)^{1 / 2}} \frac{z}{\gamma^{2}} \frac{N_{a}^{2 / 3}}{k_{B} T_{p h}}
$$

Taking into account the melting process:

$$
\left(\chi_{p h}\right)_{k}=\left(2.831 \times 10^{-13} \frac{z}{A^{1 / 2} \gamma^{2}} \frac{N_{a, \mathrm{~K}}^{2 / 3}}{T_{p h}[K]}\right)_{k}\left[\frac{\mathrm{cm}^{2}}{s}\right], k=s, l
$$

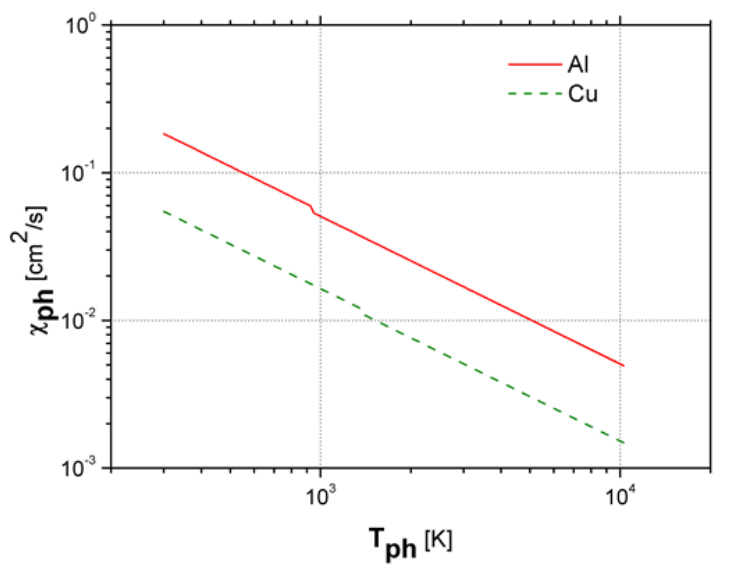

Figure 7. Temperature dependences of phonon thermal diffusivity $\chi_{p h}\left(T_{p h}\right)$.

\subsubsection{Thermal conductivity of phonon gas $\lambda_{p h}$}

The thermal conductivity of phonon gas can be expressed through its heat capacity $C_{p h}$ and thermal conductivity. Taking into account expressions for $C_{p h}$ and $\chi_{p h}$ in the hightemperature region $T_{p h}>T_{D}$ thermal conductivity takes the form:

$$
\left(\lambda_{p h}\right)_{\kappa}=C_{p h} \chi_{p h}=\left(\frac{3 \pi^{2}}{(3 m)^{3 / 2}} \frac{\hbar^{3}}{M_{0}^{1 / 2}} \frac{z}{A^{1 / 2} \gamma^{2}} \frac{N_{a}^{5 / 3}}{T_{p h}[K]}\right)_{k}=\left(1.17 \times 10^{-35} \frac{z}{A^{1 / 2} \gamma^{2}} \frac{N_{a \kappa}^{5 / 3}}{T_{p h}[K]}\right)_{\kappa}
$$


Graphic dependences of $\lambda_{p h}$ for $A l$ and $C u$ are represented in Fig. 8.

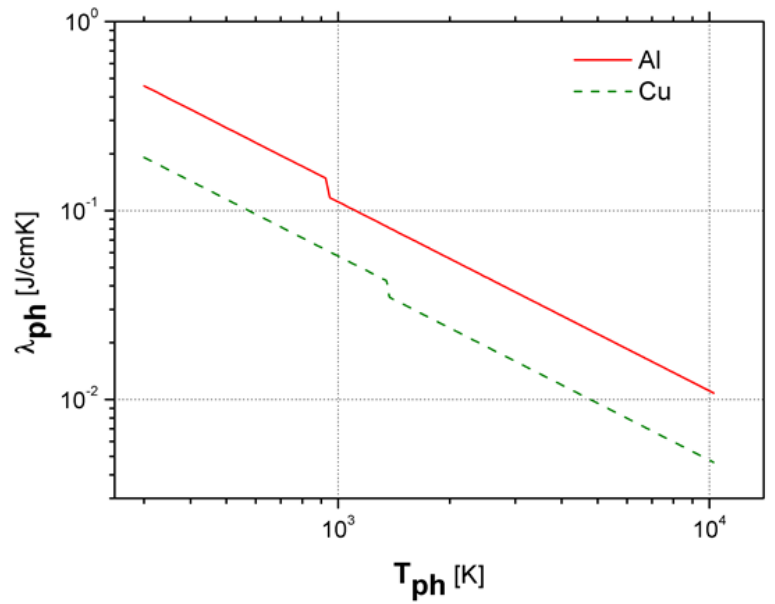

Figure 8. Temperature dependence of the phonon thermal conductivity.

\subsubsection{Equilibrium heat capacity and thermal conductivity of metal}

The temperature dependences of the total heat capacity and thermal conductivity of a metal can be represented as corresponding sums, which consist of two parts - electron (40), (51) and phonon (52), (55):

$$
\begin{gathered}
C(T)=C_{e}(T)+C_{p h}(T), \\
\lambda(T)=\lambda_{e}(T)+\lambda_{p h}(T)
\end{gathered},
$$

Temperature dependences of $\lambda(T)$ and $C(T)$ are shown in Figs 9, 10. For comparison, reference data from [72], [74] - [77] are shown by markers.

According to the equilibrium theory of metals [69], [73] in the temperature range $T_{0}<T<T_{m}$ heat transport by electrons is dominated. As predicted by the Wiedemann-Franz law, equilibrium electron thermal conductivity is practically independent of temperature. Its contribution to the total thermal conductivity of the metal with temperature increasing, is much greater than the contribution of the phonon component $\lambda_{\mathrm{e}}>\lambda_{\mathrm{ph}}$. The phonon part of thermal conductivity is inversely proportional to the temperature, and its contribution is noticeable only at low temperatures. As a result, the resulting thermal conductivity of solidstate becomes linearly decreasing temperature dependence. At the phase transition solid liquid thermal conductivity of both metals decreases abruptly in $2 \div 3$ times and decreases slowly with increasing temperature.

At very low temperatures, much smaller than Debye temperature $T<T_{D}$, these relations become invalid, which is associated with the change of interaction mechanisms. Since the 
thermal conductivity of metal bodies at low temperatures is determined by the transfer of thermal energy by sound waves, rather than electrons. The heat capacity and the number of phonons in this case are proportional to $\sim \mathrm{T}^{3}$, and the electrical resistance, defined by the scattering of electrons by phonons is inversely proportional to absolute temperature.

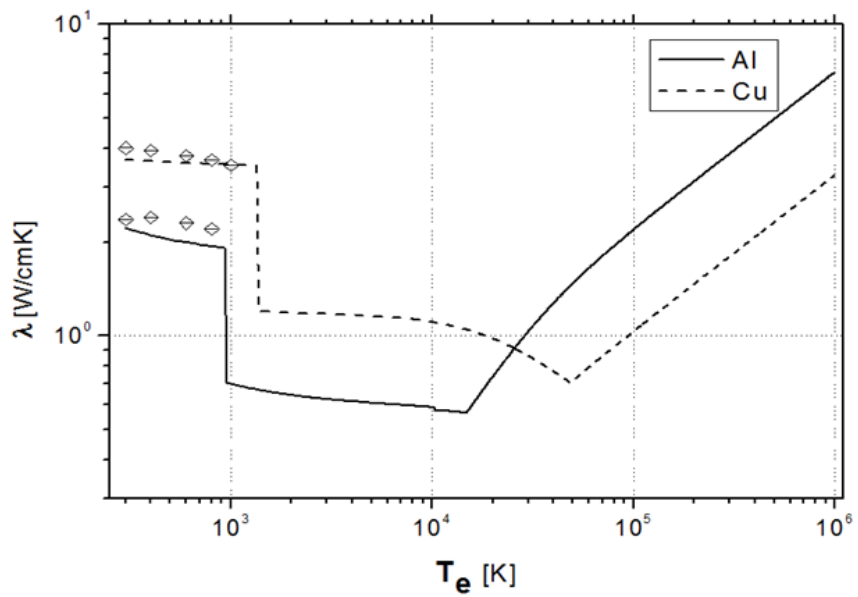

Figure 9. Temperature dependences of total thermal conductivity $\lambda(T)$.

Calculations showed that at temperatures close to the plasma $\mathrm{T} \geq 1 \mathrm{eV}$, the thermal conductivity of metals increases rapidly, and the thermal conductivity of aluminum, due to three times exceeded electron density becomes higher than the thermal conductivity of copper. However, this situation can have meaning only in the case of strongly nonequilibrium with hot electrons and cold lattice. Under equilibrium conditions, the use of calculated data should be restricted to the region of the critical point, in which neighborhood, as known, all physical properties of metals vary sharply.

Comparison of the obtained theoretical data with reference data was carried out in a relatively narrow temperature range, since the physical properties of most metals were measured in the temperature range from several tens to several thousand degrees, and usually not exceeding the boiling temperature of the equilibrium. In the range $300 \div 1500 \mathrm{~K}$, the comparison has shown a complete qualitative agreement of results with a good quantitative correlation, Figs.9, 10.

\subsection{Electron-phonon interaction}

During laser action on metals, entire pulse energy is transmitted directly to the electrons. The result is a highly nonequilibrium region in the solid with hot electrons and cold lattice. Cooling of electron subsystem realized by two mechanisms: energy transfer by electron thermal conductivity, leveling the gradients of electron temperature and electron-phonon interaction, which leads to heating of the lattice. 


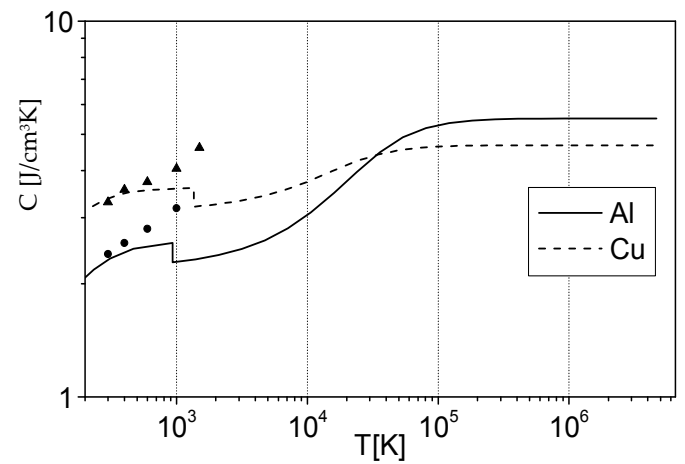

Figure 10. Temperature dependences of total heat capacity $C(T)$.

\subsubsection{The average frequency of electron-phonon collisions $\left\langle v_{\text {eph }}\right\rangle$.}

Per time unit, the electron undergoes an average of $\sim 1 / 2 \tau$ collisions with the emission of phonons, and roughly the same, but slightly smaller, the number of collisions with the absorption of phonon. Estimate the time of inelastic collisions by using the following equation [70]

$$
\frac{1}{2} \tau_{e p h}=\frac{1}{N_{e}\left\langle v_{e}\right\rangle \sigma_{e p h}} .
$$

Hence, the average frequency of electron-phonon collisions with energy transfer of electrons to crystal lattice can be represented as

$$
\left\langle v_{e p h}\right\rangle=2 N_{e}<v_{e}>\sigma_{e p h}=2 z N_{a}<v_{e}>\sigma_{e p h}
$$

where $\sigma_{e p h}$ is scattering cross section of electrons by phonons.

The cross section for electron-phonon scattering, calculated under the assumption that the product of the wave number of electron and atom displacements from equilibrium [78] has the form

$$
\sigma_{e p h}=4 \pi\left(\frac{4}{9 \pi}\right)^{4 / 3} \frac{m k_{B} T_{p h}}{\hbar^{2}}\left(\frac{r}{r_{B}}\right)^{2} \frac{r^{4}}{z^{4 / 3}} \frac{\xi \cdot\left[\ln \left(t_{1}+1\right)-\frac{t_{1}}{\left(t_{1}+1\right)}\right]}{\left(\xi^{2}+0.16\right)^{1 / 2}\left(\xi^{2}+\frac{4}{9}\right)^{1 / 2}}
$$

The cross section has a maximum at $T_{e} \approx \varepsilon_{F}$. At $T_{e}<<\varepsilon_{F}$ due to the Pauli principle, the cross section is proportional to temperature $T_{e}$. After removing the degeneracy $T_{e} \gg \varepsilon_{F}$, cross section of electron-phonon collisions decreases with $T_{e}$ increasing. The linear lattice temperature $T_{p h}$ dependence of the cross section of electron scattering in metals reflects the 
fact that the effective radius of the atoms can be identified with the amplitude of its thermal fluctuations.

Taking into account expressions for $\sigma_{e p h}$ (59) and $\left\langle v_{e}\right\rangle$ (45), the average rate of energy transfer can be written as:

$$
\left\langle v_{e p h}\right\rangle=\left(\frac{3}{2}\right)^{1 / 2} \frac{8}{3 \pi}\left(\frac{r}{r_{B}}\right)^{2} \frac{k_{B}}{\hbar} T_{p h} \Phi_{e p h}(\xi) \cdot\left\{\begin{array}{c}
\Phi_{\text {eph }}(\xi)=\frac{\xi \cdot\left[\ln \left(t_{1}+1\right)-\frac{t_{1}}{\left(t_{1}+1\right)}\right]}{\left(\xi^{2}+0.16\right)^{1 / 4}\left(\xi^{2}+\frac{4}{9}\right)^{1 / 2}} \text { при } \quad \xi<1 \\
\Phi_{\text {eph }}(\xi)=\frac{\xi \cdot\left[\ln \left(t_{1}+1\right)-\frac{t_{1}}{\left(t_{1}+1\right)}\right]}{\left(\xi^{2}+0.16\right)^{3 / 4}\left(\xi^{2}+\frac{4}{9}\right)^{1 / 2}} n p u \quad \xi \geq 1
\end{array}\right\}
$$

The dependences of the mean frequency of transmission electron energy to phonons from the temperature $T_{e}$ at $T_{p h}=300 \mathrm{~K}$ and $T_{p h}=1000$ for aluminum and copper are shown in Fig.11.

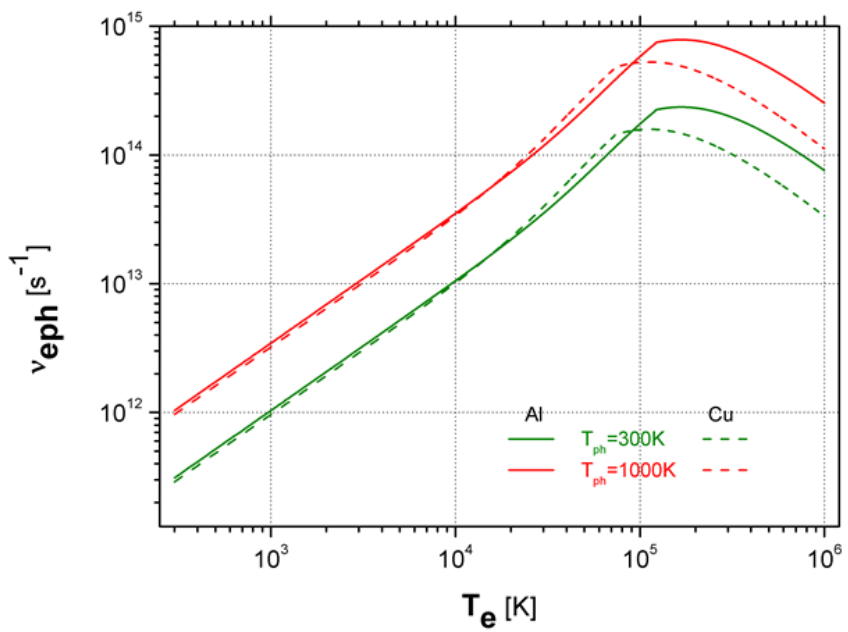

Figure 11. Temperature dependences of frequency of energy transfer of electrons to phonons at $300 \mathrm{~K}$ and $=1000 \mathrm{~K}$.

\subsubsection{Energy transfer}

The mechanism of energy transfer to the lattice due to electron-phonon collisions was discussed in several papers [79-81]. The collision of an electron with the lattice phonon is absorbed or is born, for which law of conservation of energy and momentum works: 


$$
\begin{gathered}
\Delta \varepsilon=m v_{1}^{2} / 2-m v_{2}^{2} / 2= \pm \hbar \Omega \\
p=2 m v_{F} \sin \frac{\varphi}{2} \cong \frac{\hbar \Omega}{v_{\text {sound }}}
\end{gathered}
$$

where $\Omega$ - the phonon frequency, $\varphi$ - the angle between the initial and final electron momentum $m v_{1}$ and $m v_{2}, v_{\text {sound }}$ - velocity of sound, $p$ - the electron momentum.

From the conservation laws (61) it follows that energy of phonon, excited by an electron with a momentum $p$ equal to $\Delta \varepsilon=\hbar \Omega=2 p v_{\text {sound }} \sin \frac{\varphi}{2}$.

At a spherically symmetric scattering the energy transferred by one electron per unit time, averaged by $\varphi$, equals [81]:

$<\Delta \varepsilon>=\frac{2 \hbar \Omega_{D}}{3 k_{B} T_{p h}} p v_{\text {sound }} v_{\text {eph }}\left[1-\frac{1}{32}\left(\frac{\hbar \Omega_{D}}{p v_{\text {sound }}}\right)^{3}\right]$, where $\hbar \Omega_{D}$ - the Debye energy.

Taking into account velocity of sound $v_{\text {sound }}$, expressed in terms of the Fermi velocity [73], $<\Delta \varepsilon>$ in its final form is written:

$$
\begin{aligned}
& <\Delta \varepsilon>=\frac{2}{3} p_{F} v_{\text {sound }}^{2} \frac{\hbar k_{D}}{k_{B} T_{p h}}\left[1-\frac{1}{32}\left(\frac{\hbar k_{D} v_{\text {sound }}}{p_{F} v_{\text {sound }}}\right)^{3}\right] \frac{v_{\text {eph }}}{k_{B} T_{p h}}= \\
& =\left(\frac{9 \pi}{2}\right)^{1 / 3}\left(\frac{3}{2}\right)^{1 / 2} \frac{4}{3} \frac{z}{m M}\left(\frac{r}{r_{B}}\right)^{2} \frac{\hbar^{3}}{r^{4}} \times\left[1-\left(\frac{1}{2^{4} z}\right)\right] \Phi_{\text {eph }}(\xi),
\end{aligned}
$$

\subsubsection{The electron-fonon coupling factor}

The average energy transferred to phonons by electrons per volume unit per time unit is equal to the product of the average energy transferred by a single electron $\langle\Delta \varepsilon\rangle$ and concentration of electrons, which may participate in the transfer of energy to phonons

$$
\frac{d \varepsilon}{d t}= \begin{cases}<\Delta \varepsilon>\frac{k_{B}\left(T_{e}-T_{p h}\right)}{\varepsilon_{F}} N_{e} & T_{e}<\varepsilon_{F} \\ <\Delta \varepsilon>z\left(T_{e}\right) N_{e} & T_{e} \geq \varepsilon_{F}\end{cases}
$$

or

$$
\frac{d \varepsilon}{d t}=<\Delta \varepsilon>\frac{k_{B}\left(T_{e}-T_{p h}\right)}{\varepsilon_{F}} N_{e}=g(\xi)\left(T_{e}-T_{p h}\right),
$$

where $g(\xi)$ is electron-fonon coupling factor, equal to 


$$
g(\xi)=\langle\Delta \varepsilon\rangle \frac{k_{B} N_{e}}{\varepsilon_{F}}=\left(\frac{3}{2}\right)^{1 / 2}\left(\frac{8}{9 \pi}\right)^{1 / 3} \frac{8}{3} \cdot \frac{\hbar k_{B}}{r_{B}^{2} M_{0}} \frac{z^{2}}{A} N_{a}\left[1-\left(\frac{1}{2^{4} z}\right)\right] \Phi_{e p h}(\xi),\left[\frac{\mathrm{W}}{m^{3} K}\right] .
$$

The temperature dependence of the electron-fonon coupling factor $g\left(T_{e}\right)$ is shown in Fig.12. The results calculated for $A l$ in [82] are shown to compare by markers.

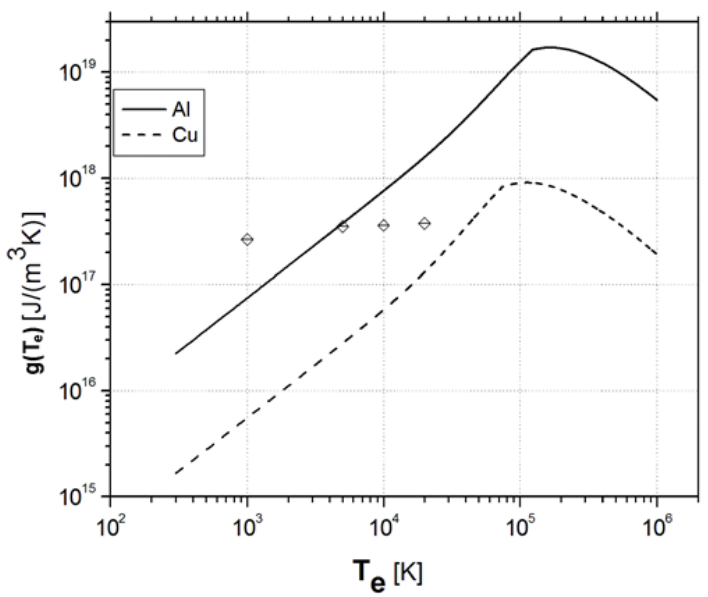

Figure 12. Temperature dependences of electron-fonon coupling factor $g\left(T_{e}\right)$.

\subsection{Conclusions}

Important thermodynamic and thermophysical properties of a degenerate electron and phonon gas: equation of state, heat capacity, the energy exchange between subsystems in an arbitrary temperature range are determined using fundamental physical quantities - the mean free paths and times (frequency) of the electron - electron and electron-phonon collisions.

Using Fermi integrals technique and its subsequent approximations have provided the temperature dependence of all characteristics in the form of simple analytical expressions.

\section{Formulation and solution of the problem of laser action on metals using the method of dynamic adaptation}

Action of intense laser radiation on metals may be accompanied by heating, evaporation, plasma formation. The dynamics of the processes in the condensed media and in the flow of vapor and gas environment surrounding the evaporating surface depend on several parameters of the laser pulse: the level of the absorbed intensity, wavelength, duration and spatial and temporal distribution of the energy, as well as optical, thermal and hydrodynamic characteristics of the condensed and gaseous media. It should be noted that the process of interaction is qualitatively different depending on where the target is placed: in the vacuum or the gaseous medium. 
As it was already mentioned, the main features of ultra-short super-power action on metals are associated with the high speed and voluminous nature of the laser pulse energy release. The high rate of energy input leads to a strong deviation of the system from the local thermodynamic equilibrium (LTE). The basis of the mathematical description of the pulsed laser heating of metals is a phenomenological two-temperature model (TTM) of parabolic type proposed in the 50s by the authors of [65], [66]. The application of TTM involves the description of the situations with small deviations from LTE. Ultrafast heating of metal targets by super-power laser pulses causes a strong deviation from the LTE and requires appropriate adjustments of the mathematical model. In the first place, the kinetic nonequilibrium phase transitions and powerful dynamic effects associated with the movement of the phase fronts must be taken into account. The consideration of these processes requires the explicit description of the kinetics of the phase transitions and the formulation of the conservation laws at the phase fronts, which are hydrodynamic breaks.

\subsection{Mathematical description of processes in the irradiation zone}

Laser radiation propagates from the right to the left and strikes the surface of the metal target. Then the radiation is partially absorbed and partially reflected. The absorbed energy is consumed for heating, phase transformations and the generation of shock waves in the solid phase. Fig.13 shows spatial location of phases, moving interphase boundaries $\Gamma_{s \ell}(t)$, $\Gamma_{\ell v}(t)$ and shock wave in solid $\Gamma_{s h, s}(t)$.

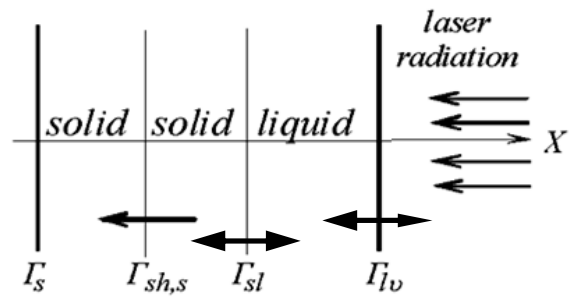

Figure 13. Spatial phase configuration.

Formulation of the problem was carried out under the following assumptions and limitations:

- The mechanisms of volume of melting and evaporation are not included into the consideration.

- The melting and evaporation fronts appear at the irradiated surface during its noticeable overheating $\Delta T_{s \ell}=T_{\text {sur }}-T_{m}$ and $\Delta T_{\ell v}=T_{\text {sur }}-T_{b}\left(p_{\text {sat }}\right)$, the overheated metastable states act sustainably during the consideration.

The mathematical description and modeling of pico- and femtosecond laser action on metal target in vacuum was performed within the framework of a two-temperature and spatially one-dimensional multi-front nonequilibrium hydrodynamic Stefan problem, written for the two phases - solid and liquid. 


\subsubsection{System of equations}

Action of high-power laser pulses on metal targets initiate in them a number of interrelated processes with nonlinear behavior. The description of their behavior is performed using a system of hydrodynamic equations, two energy balance equations for electron and phonon subsystems, and the transport equation of the laser radiation. The system of equations can be written in the domain with three moving boundaries $\Gamma_{s h, s}(t), \Gamma_{s \ell}(t), \Gamma_{k v}(t)$.

$$
\begin{aligned}
& \left(\begin{array}{l}
\frac{\partial \rho}{\partial t}+\frac{\partial(\rho u)}{\partial x}=0 \\
\frac{\partial(\rho u)}{\partial t}+\frac{\partial\left(\rho u^{2}\right)}{\partial x}+\frac{\partial p}{\partial x}=0 \\
\frac{\partial\left(\rho_{e} \varepsilon_{e}\right)}{\partial t}+\frac{\partial\left(\rho_{e} u \varepsilon_{e}\right)}{\partial x}=-\left(p \frac{\partial u}{\partial x}+\frac{\partial W_{e}}{\partial x}+g\left(T_{e}\right)\left(T_{e}-T_{p h}\right)+\frac{\partial G}{\partial x}\right) \\
\frac{\partial\left(\rho \varepsilon_{p h}\right)}{\partial t}+\frac{\partial\left(\rho u \varepsilon_{p h}\right)}{\partial x}=-\left(p \frac{\partial u}{\partial x}+\frac{\partial W_{p h}}{\partial x}-g\left(T_{e}\right)\left(T_{e}-T_{p h}\right)\right) \\
\frac{\partial G}{\partial x}+\alpha\left(T_{e}\right) G=0, \quad \rho_{e}=z \frac{m}{M} \rho, \\
P=P_{e}\left(\rho_{e}, T_{e}\right)+P_{p h}\left(\rho, T_{p h}\right), \quad \varepsilon_{e}=C_{e}\left(T_{e}\right) T_{e}, \quad \varepsilon_{p h}=C_{p h}\left(T_{p h}\right) T_{p h}
\end{array}\right)_{k=s, \ell} \\
& t>0, \quad \Gamma_{s}<x<\Gamma_{s h, s}(t) \cup \Gamma_{s h, s}(t)<x<\Gamma_{s \ell}(t) \cup \Gamma_{s \ell}(t)<x<\Gamma_{\ell v}(t) \quad, \\
& W_{e}=-\lambda\left(T_{e}, T_{p h}\right) \frac{\partial T_{e}}{\partial x}, W_{p h}=-\lambda\left(T_{p h}\right) \frac{\partial T_{p h}}{\partial x}, P(\rho, T)=P\left(\rho_{e}, T_{e}\right)+P\left(\rho, T_{p h}\right) \text {, }
\end{aligned}
$$

the designations: $\rho, u, \varepsilon, T, p$ are the density, gas-dynamic velocity, internal energy, temperature and pressure correspondingly, $\alpha\left(T_{e}\right), R\left(T_{e}\right)$ are the coefficient of volume absorption of the laser radiation and surface reflectivity, $G$ is the density of the laser radiation, $C_{e}\left(T_{e}\right), C_{p h}\left(T_{p h}\right), \lambda_{e}\left(T_{e}, T_{p h}\right), \lambda_{p h}\left(T_{p h}\right)$ are electron and phonon heat capacity and heat conductivity, $g\left(T_{e}\right)$ electron-phonon coupling factor. Indexes $s, \ell, v$, designate solid, liquid, vapor phases, $e, p h$ designate electron and phonon components.

\subsubsection{Edge conditions}

We use edge conditions in the form of initial conditions at $t=0$ and boundary conditions at $x=\Gamma_{s}, \Gamma_{s h, s}(t), \Gamma_{s \ell}(t), \Gamma_{\ell v}(t)$.

\section{Initial conditions}

$$
t=0: \quad u(0, x)=0, \quad p=0, \quad \rho=\rho_{0}, \quad T_{e}=T_{p h}=T_{0}=293 \mathrm{~K}
$$

\section{Boundary conditions}

$x=\Gamma_{s}$ : the condition of zero mass and heat flow is used as boundary conditions at the left (fixed) boundary: 


$$
x=\Gamma_{s}: \rho_{s} u_{s}=0, \quad W_{T}=0 ;
$$

$x=\Gamma_{s \ell}(t)$ : the model of surface melting-crystallization is used as boundary conditions at the moving interphase boundary $\Gamma_{s \ell}(t)$.

The model of surface melting-crystallization is a non-equilibrium kinetic version of the Stefan problem [18], [83,84], formulated for the conditions of significant deviation from LTE and consisting of three conservation laws: mass, momentum and energy, written in the stationary (laboratory) coordinate system:

$$
\begin{aligned}
& x=\Gamma_{s \ell}(t): \rho_{s}\left(u_{s}-v_{s \ell}\right)=\rho_{\ell}\left(u_{\ell}-v_{s \ell}\right) \\
& P_{s}+\rho_{s}\left(u_{s}-v_{s \ell}\right)^{2}=P_{\ell}+\rho_{\ell}\left(u_{\ell}-v_{s \ell}\right)^{2}, \\
& \left(\lambda_{p h} \frac{\partial T_{p h}}{\partial x}\right)_{s}-\left(\lambda_{p h} \frac{\partial T_{p h}}{\partial x}\right)_{\ell}=\rho_{s} L_{m}^{n e} v_{s \ell},
\end{aligned}
$$

These conservation laws are accompanied by the kinetic dependence of the interphase front velocity $v_{s \ell}\left(\Delta T_{s \ell}\right)$ on the overheating of the solid surface (22).

$$
v_{s \ell}\left(T_{s \ell}\right)=\frac{a f}{\lambda}\left(3 k_{B} T_{s \ell} / m\right)^{1 / 2}\left[1-\exp \left(\frac{L_{m}}{k_{B}}\left(\frac{\Delta T}{T_{s \ell}}\right)\right)\right],
$$

Additional account of hydrodynamic effects was carried out using the expressions for the curve of equilibrium melting $T_{m}\left(P_{s}\right)$, temperature dependence of the equilibrium latent melting heat $L_{m}^{e q}\left(T_{m}\left(P_{\mathrm{s}}\right)\right)$ and non-equilibrium latent melting heat $L_{m}^{\text {ne }}$

$$
L_{m}^{n e}=L_{m}^{e q}\left(T_{m}\left(P_{\mathrm{s}}\right)\right)+\Delta C_{p s \ell} \Delta T_{s \ell}+\frac{\rho_{s}+\rho_{\ell}}{\rho_{s}-\rho_{\ell}} \frac{\left(u_{s}-u_{\ell}\right)^{2}}{2}, T_{m}=T_{m}\left(P_{\mathrm{s}}\right)=T_{m, 0}+k P_{\mathrm{s}},
$$

where $\Delta C_{p s \ell}=\left(C_{p \ell}-C_{p s}\right), \Delta T_{s \ell}=\left(T_{s \ell}-T_{m}\left(P_{s}\right)\right)$.

The electron component is assumed continuous with respect to the electron density $N_{e}$ and temperature $T_{e}$ during transition through the phase front:

$$
\left(\lambda_{e} \frac{\partial T_{e}}{\partial x}\right)_{s}=\left(\lambda_{e} \frac{\partial T_{e}}{\partial x}\right)_{\ell}, \quad T_{e, s}=T_{e, \ell}
$$

$x=\Gamma_{k v}(t)$ : the model of surface evaporation is used as boundary conditions at the moving interphase boundary $\Gamma_{k v}(t)$. The process of surface evaporation within the approximation of the Knudsen layer is described by three conservation laws and three additional parameters at the outer side of the Knudsen layer (temperature $T_{v}$, density $\rho_{v}$ and velocity $u_{v}$ ). In the general case, two of these parameters (usually $T_{v}$ and $\rho_{v}$ ) are determined using 
certain approximation relations, e.g. (30), while the third one (usually the Mach number $M=u\left(u_{c}\right)$ is found from the solution of the gas-dynamic equations.

Three conservation laws are written in the stationary (laboratory) coordinate system and have the form:

$$
\begin{aligned}
& x=\Gamma_{k v}(t): \rho_{k}\left(u_{k}-v_{k v}\right)=\rho_{v}\left(u_{v}-v_{k v}\right), \\
& p_{k}+\rho_{k}\left(u_{k}-v_{k v}\right)^{2}=p_{v}+\rho_{v}\left(u_{v}-v_{k v}\right)^{2}, \\
& \left(\lambda_{p h} \frac{\partial T_{p h}}{\partial x}\right)_{k}-\left(\lambda_{v} \frac{\partial T_{v}}{\partial x}\right)_{v}=\rho_{k}\left(u_{k}-v_{k v}\right) L_{v}^{n e} \\
& \rho_{v}=\alpha_{\rho}(M) \rho_{\text {sat }}, T_{v}=\alpha_{T}(M) T_{\text {sur }}, \\
& p_{\text {sat }}\left(T_{\text {sur }}\right)=p_{b} \exp \left[\frac{L_{v}^{n e}}{R T_{b}}\left(\frac{\Delta T_{\text {sur }}}{T_{\text {sur }}}\right)\right], \rho_{\text {sat }}=\frac{p_{\text {sat }}\left(T_{\text {sur }}\right)}{R T_{\text {sur }}}, \Delta T_{\text {sur }}=T_{\text {sur }}-T_{b}\left(p_{\text {sat }}\left(T_{\text {sur }}\right)\right) \\
& L_{v}^{n e}=L_{v}^{e q}\left(T_{\text {sur }}\right)+C_{p v}\left(T_{v}-T_{\text {sur }}\right)+\frac{\rho_{k}+\rho_{v}}{\rho_{k}-\rho_{v}} \frac{\left(u_{k}-u_{v}\right)^{2}}{2},
\end{aligned}
$$

The boundary conditions for the electron component and the laser radiation are written as:

$$
\begin{gathered}
-\lambda_{e} \frac{\partial T_{e}}{\partial x}=\sigma T_{e}^{4}, \\
G(t)=\left(1-R_{k}\left(T_{e}\right)\right) \cdot G_{0} \exp \left(-\left(\frac{t}{\tau_{L}}\right)^{2}\right) .
\end{gathered}
$$

$\sigma$ is the Stefan-Boltzmann constant.

$x=\Gamma_{\text {sh,s }}(t)$ : three conservation laws are written at the moving front of the shock wave, socalled Rankine-Hugoniot relations, that have the following form in the laboratory coordinate system:

$$
\begin{gathered}
j_{s h, s}^{m}=\rho_{1}\left(u_{1}-v_{s h, s}\right)=\rho_{0}\left(u_{0}-v_{s h, s}\right), \\
j_{s h, s}^{i}=p_{1}+\rho_{1}\left(u_{1}-v_{s h, s}\right)^{2}=p_{0}+\rho_{0}\left(u_{0}-v_{s h, s}\right)^{2}, \\
j_{s h, s}^{e}=-W_{T, 1}+j_{s h, s}^{m}\left[\varepsilon_{1}+\frac{\left(u_{1}-v_{s h, s}\right)^{2}}{2}\right]=-W_{T, 0}+j_{s h, s}^{m}\left[\varepsilon_{1}+\frac{\left(u_{0}-v_{s h, s}\right)^{2}}{2}\right]
\end{gathered}
$$

The index " 0 " signifies the values at the background side, " 1 " relates to the shock wave side. 


\subsection{Numerical algorithm and finite difference schemes}

Mathematical feature of Stefan problems is the lack of explicit expressions for the interconnected quantities at the interphase boundaries: temperature $T_{s \ell}, T_{\text {sur }}$ and velocities of the phase fronts $v_{s \ell}$ and $v_{k v}$. So the problem (65) - (81) will be nonlinear even for constant values of thermo-physical and optical properties. For low velocities of phase transformations ( $\left.v_{s \ell}, v_{k v}<<v_{\text {sound }}\right)$, the processes behave in a quasi-equilibrium way, and their description can be performed within the framework of equilibrium models. For example, in the problem of melting-crystallization, a phenomenological condition of the temperature equity $T_{s \ell}=T_{s}=T_{\ell}=T_{m}$ is used instead of the kinetic condition (71), and the differential Stefan condition is completely omitted. The influence of the phase transition is taken into account using a singular adding of the latent heat $L_{m}^{e q}$ to the heat capacity (equation of state) in the point of the phase transition, so-called enthalpy method [85 - 87]. To solve the problems in this statement, the methods of the type of "pass-through" or uniform schemes have found wide application [85], where the velocity and location of the phase transition are not determined explicitly.

Fast phase transitions $\left(v_{s \ell}, v_{k v}<<v_{\text {sound }}\right)$, that are typical for powerful pulsed laser action, occur under the conditions of high non-equilibrium caused by the powerful flow of material [88] over the interphase boundary. Using the smoothing procedure of the enthalpy reduces the class of solutions of the phase transformations problems in the material; in particular, it excludes from consideration the effects of overheating and overcooling of the condensed matter [89, 90]. High-speed phase transitions require explicit tracking of the phase boundaries [91]. Typically, the velocity of phase boundaries is determined numerically. In computational respect, the presence of moving boundaries leads to a significant complication of the numerical solution [92].

\subsubsection{Method of dynamic adaptation}

Finite-difference method of dynamic adaptation [93], [94], was used to numerically solve the discussed problem. This method allows performing explicit tracking of any number of interphase boundaries and shock waves [95].

The method of dynamic adaptation is based on the procedure of transition to an arbitrary nonstationary coordinate system. Its usage allows to formulate the problem of grid generation and adaptation on a differential level, i.e. part of differential equations in the obtained mathematical model describes physical processes, and other part describes the behavior of the grid nodes [96].

\subsection{Arbitrary nonstationary coordinate system}

The transition to an arbitrary nonstationary coordinate system is performed using an automatic coordinate transformation using the sought solution. Formally, it is possible to use a variable change of the common form $x=f(q, \tau), t=\tau$ to perform a transition from the 
physical space $\Omega_{\mathrm{x}, \mathrm{t}}$ with Euler variables $(x, t)$ to some computational space with arbitrary nonstationary coordinate system $\Omega_{q, \tau}$ and variables $(q, \tau)$. It is assumed that this transformation corresponds to a univalent nondegenerate reverse transformation $q=\phi(x, t), \quad \tau=\mathrm{t}$. During the transition between coordinate systems, the partial derivatives of the dependent variables are connected using the following expressions:

$$
\frac{\partial}{\partial t}=\frac{\partial}{\partial \tau}+Q \frac{\partial}{\partial q}, \frac{\partial}{\partial x}=\frac{1}{\partial \psi} \frac{\partial}{\partial q}, \frac{\partial^{2}}{\partial x^{2}}=\frac{1}{\psi} \frac{\partial}{\partial q} \frac{1}{\psi} \frac{\partial}{\partial q},
$$

where $\psi=\partial x / \partial q$ is the Jacobian of the reverse transformation, $Q$ is the transformation function that should be determined.

In the arbitrary coordinate system, moving with velocity $Q$, where $\frac{\partial x}{\partial \tau}=-Q$, or $\frac{\partial \psi}{\partial \tau}=-\frac{\partial Q}{\partial q}$, the differential model $(65)$ in the new variables $(q, \tau)$ takes the form:

$$
\begin{gathered}
\frac{\partial \psi}{\partial \tau}=-\frac{\partial Q}{\partial q}, \psi=\frac{\partial x}{\partial q} \\
\frac{\partial}{\partial \tau}(\psi \rho)+\frac{\partial}{\partial q}(\rho(u+Q))=0 \\
\frac{\partial}{\partial \tau}(\psi \rho u)+\frac{\partial}{\partial q}(p+\rho u(u+Q))=0 \\
\frac{\partial}{\partial \tau}\left(\psi \rho_{e} \varepsilon_{e}\right)+\frac{\partial}{\partial q}\left(\rho_{e} \varepsilon_{e}(u+Q)\right)=-\left(p \frac{\partial u}{\partial q}+\frac{\partial W_{e}}{\partial q}+g\left(T_{e}\right)\left(T_{e}-T_{p h}\right)+\frac{\partial G}{\partial q}\right) \\
\frac{\partial\left(\psi \rho \varepsilon_{p h}\right)}{\partial \tau}+\frac{\partial}{\partial q}\left(\rho \varepsilon_{p h}(u+Q)\right)=-\left(p \frac{\partial u}{\partial q}+\frac{\partial W_{p h}}{\partial q}-g\left(T_{e}\right)\left(T_{e}-T_{p h}\right)\right) \\
\frac{\partial G}{\partial q}+\psi \alpha\left(T_{e}\right) G=0 \\
p=p_{e}\left(\rho_{e}, T_{e}\right)+p_{p h}\left(\rho, T_{p h}\right), \quad \varepsilon_{e}=C_{e}\left(T_{e}\right) T_{e}, \quad \varepsilon_{p h}=C_{p h}\left(T_{p h}\right) T_{p h}, \quad W=-\frac{\lambda(T)}{\psi} \frac{\partial T}{\partial q}
\end{gathered}
$$

Thus, usage of an arbitrary nonstationary coordinate system is accompanied by the transformation of the initial differential model (65) into the extended model (83) - (88), in which the equation (83) is the equation of the inverse transform. Equations (84) - (88) describes the physical processes. The type, characteristics and kind of boundary conditions 
for the inverse transformation equations depend on the type conversion function $Q$. Because of this equation, the initial (66) and boundary conditions (67) - (81) are changed accordingly.

Thus, the unknown functions in the computational space are not only the functions of the physical fields but also the coordinates of the grid nodes. The equation of the reverse transformation is used after the determination of the function $Q$ to construct the adaptive grid in the physical space. Its differential analogue describes the dynamics of the grid nodes, and the function $Q$ performs controlled movement of the nodes in agreement with the dynamics of the sought solution. In the computational space, the nodes of the grid and all discontinuous and interphase boundaries are steady. The value of the function $Q$ at the boundaries of the domain are determined from the boundary conditions.

Selection of the function $Q$. The function $Q$ that is in agreement with the sought solution can be determined from the quasi-steady principle [96], which states that we should search for such nonstationary coordinate system where all processes occur in a steady way. Since the energy balance equation includes all hydrodynamic variables, to determine the function $Q$, we can use only two equations (86), (87), assuming that $\frac{\partial \varepsilon_{e}}{\partial \tau}=\frac{\partial \varepsilon_{p h}}{\partial \tau}=0$.Then the function $Q$ will have the form

$$
Q=-u-\frac{2 p \frac{\partial u}{\partial q}+\left(+\frac{\partial W_{e}}{\partial q}+\frac{\partial W_{p h}}{\partial q}+\frac{\partial G}{\partial q}\right)}{\left(\rho_{e} \frac{\partial \varepsilon_{e}}{\partial q}+\rho \frac{\partial \varepsilon_{p h}}{\partial q}+r e\right)}
$$

where $W_{e}=-\frac{\lambda_{e}}{\Psi} \frac{\partial T_{e}}{\partial q}, W_{p h}=-\frac{\lambda_{p h}}{\Psi} \frac{\partial T_{p h}}{\partial q}$.

\section{Differential schemes}

The differential model (83) - (88) was approximated by a family of conservative differential schemes obtained using integration-interpolation method [97]. Computational grids $\left(\omega_{m}^{j}\right)_{s}$ and $\left(\omega_{m}^{j}\right)$ are introduced in the computational space $\Omega_{q, \tau}$ in each subdomain with integer $q_{i}$ and half-integer $q_{i+1 / 2}$ nodes for the spatial variable $q$ and step $\Delta \tau^{j}$ for the variable $\tau$.

$$
\left(\omega_{m}^{j}\right)_{k}=\left\{\begin{array}{c}
\left(q_{m}, \tau^{j}\right),\left(q_{m+1 / 2}, \tau^{j}\right) ; \quad q_{m+1}=q_{m}+h, \quad q_{m+1 / 2}=q_{m}+0.5 h, \\
\tau^{j+1}=\tau^{j}+\Delta \tau^{j}, \quad i=0, . ., N-1, \quad j=0,1, \ldots
\end{array}\right\}_{k=s, \ell}
$$

The flow variables $W_{e,}, W_{p h}, G$, and functions $u, Q, x$, correspond to the integer nodes during approximation, while functions $\varepsilon_{e}, \varepsilon_{p h}, T_{e}, T_{p h}, \rho, \psi$ correspond to half-integers ones $\left(q_{m+1 / 2}, \tau^{j}\right)$. 
The family of conservative differential schemes has the form:

$$
\begin{aligned}
& \frac{\psi_{m-1 / 2}^{j+1}-\psi_{m-1 / 2}^{j}}{\Delta \tau^{j}}=\frac{Q_{m}^{\sigma_{1}}-Q_{m-1}^{\sigma_{1}}}{h} \\
& \frac{\psi_{m-1 / 2}^{j+1} \rho_{m-1 / 2}^{j+1}-\psi_{m-1 / 2}^{j} \rho_{m-1 / 2}^{j}}{\Delta \tau^{j}}=\frac{\rho_{m}^{\sigma_{2}}\left(Q_{m}^{\sigma_{1}}-u_{m}^{\sigma_{3}}\right)-\rho_{m-1}^{\sigma_{2}}\left(Q_{m-1}^{\sigma_{1}}-u_{m-1}^{\sigma_{3}}\right)}{h} \\
& \frac{u_{m}^{j+1}\left(\psi_{m-1 / 2}^{j+1} \rho_{m-1 / 2}^{j+1}+\psi_{m+1 / 2}^{j+1} \rho_{m+1 / 2}^{j+1}\right)-u_{m}^{j}\left(\psi_{m-1 / 2}^{j} \rho_{m-1 / 2}^{j}+\psi_{m+1 / 2}^{j} \rho_{m+1 / 2}^{j}\right)}{2 \Delta \tau^{j}}=\frac{p_{m-1 / 2}^{\sigma_{4}}-p_{m+1 / 2}^{\sigma_{4}}}{h}+ \\
& +\frac{\rho_{m}^{\sigma_{2}}\left(Q_{m}^{\sigma_{1}}-u_{m}^{\sigma_{3}}\right) u_{m+1}^{\sigma_{3}}+\rho_{m+1}^{\sigma_{2}}\left(Q_{m+1}^{\sigma_{1}}-u_{m}^{\sigma_{3}}\right) u_{m}^{\sigma_{3}}}{2 h}-\frac{\rho_{m}^{\sigma_{2}}\left(Q_{m}^{\sigma_{1}}-u_{m}^{\sigma_{3}}\right) u_{m-1}^{\sigma_{3}}+\rho_{m-1}^{\sigma_{2}}\left(Q_{m-1}^{\sigma_{1}}-u_{m-1}^{\sigma_{3}}\right) u_{m}^{\sigma_{3}}}{2 h} \\
& \frac{\left(\psi_{m-1 / 2}^{j+1} \rho_{e, m-1 / 2}^{j+1} \varepsilon_{e, m-1 / 2}^{j+1}\right)-\left(\psi_{m-1 / 2}^{j} \rho_{e, m-1 / 2}^{j} \varepsilon_{e, m-1 / 2}^{j}\right)}{\Delta \tau^{j}}=-\left\{\frac{\left[\rho_{e, m}^{\sigma_{2}} \varepsilon_{e, m}^{\sigma_{5}}\left(Q_{m}^{\sigma_{1}}-u_{m}^{\sigma_{3}}\right)-\rho_{e, m-1}^{\sigma_{2}} \varepsilon_{e, m-1}^{\sigma_{5}}\left(Q_{m-1}^{\sigma_{1}}-u_{m-1}^{\sigma_{3}}\right)\right]}{h}+\right. \\
& +\frac{p_{m-1 / 2}^{\sigma_{4}}\left(u_{m-1}^{\sigma_{3}}-u_{m}^{\sigma_{3}}\right)}{h}+\frac{2\left[\lambda_{m}^{\sigma_{6}} \frac{T_{m+1 / 2}^{\sigma_{7}}-T_{m-1 / 2}^{\sigma_{7}}}{\psi_{m+1 / 2}^{\sigma_{8}}+\psi_{m-1 / 2}^{\sigma_{8}}}-\lambda_{m-1}^{\sigma_{6}} \frac{T_{m-1 / 2}^{\sigma_{7}}-T_{m-3 / 2}^{\sigma_{7}}}{\psi_{m-1 / 2}^{\sigma_{8}}+\psi_{m-3 / 2}^{\sigma_{8}}}\right]}{h}+\left[g\left(T_{e}\right)^{\sigma_{9}}\left(T_{e}-T_{p h}\right)^{\sigma_{10}}\right]_{m-1 / 2}+ \\
& \left.+\frac{\left(G_{m}^{\sigma_{11}}-G_{m-1}^{\sigma_{11}}\right)}{h}+\frac{\left(W_{e, m}^{\sigma_{12}}-W_{e, m-1}^{\sigma_{12}}\right)}{h}\right\} \\
& \frac{\left(\psi_{m-1 / 2}^{j+1} \rho_{m-1 / 2}^{j+1} \varepsilon_{p h, m-1 / 2}^{j+1}\right)-\left(\psi_{m-1 / 2}^{j} \rho_{m-1 / 2}^{j} \varepsilon_{p h, m-1 / 2}^{j}\right)}{\Delta \tau^{j}}= \\
& -\left\{\frac{\left[\rho_{m}^{\sigma_{2}} \varepsilon_{p h, m}^{\sigma_{13}}\left(Q_{m}^{\sigma_{1}}-u_{m}^{\sigma_{3}}\right)-\rho_{m-1}^{\sigma_{2}} \varepsilon_{p h, m-1}^{\sigma_{13}}\left(Q_{m-1}^{\sigma_{1}}-u_{m-1}^{\sigma_{3}}\right)\right]}{h}+\frac{p_{m-1 / 2}^{\sigma_{4}}\left(u_{m-1}^{\sigma_{3}}-u_{m}^{\sigma_{3}}\right)}{h}+\right. \\
& +\frac{2\left[\lambda_{p h, m}^{\sigma_{14}} \frac{T_{p h, m+1 / 2}^{\sigma_{15}}-T_{p h, m-1 / 2}^{\sigma_{15}}}{\psi_{m+1 / 2}^{\sigma_{8}}+\psi_{m-1 / 2}^{\sigma_{8}}}-\lambda_{p h, m-1}^{\sigma_{14}} \frac{T_{p h, m-1 / 2}^{\sigma_{15}}-T_{p h, m-3 / 2}^{\sigma_{15}}}{\psi_{m-1 / 2}^{\sigma_{8}}+\psi_{m-3 / 2}^{\sigma_{8}}}\right]}{h}+ \\
& \left.+\left[g\left(T_{e}\right)^{\sigma_{9}}\left(T_{e}-T_{p h}\right)^{\sigma_{10}}\right]_{m-1 / 2}+\frac{\left(W_{e, m}^{\sigma_{16}}-W_{e, m-1}^{\sigma_{16}}\right)}{h}\right\}
\end{aligned}
$$

Here $f^{\sigma_{r}}=\sigma_{r} f^{j+1}+\left(1-\sigma_{r}\right) f^{j}$, and $\sigma_{r}=\sigma_{1}, \sigma_{2}, \ldots$ aare the weight coefficients, determining the degree of implicitness of the differential schemes.

To solve the obtained system of nonlinear partial-differential equations we use a computational algorithm with enclosed iterative cycles consisting of one external and two internal cycles [98]. Each of the internal cycles uses the Newton iterative procedure. 


\section{Modeling results}

We consider two modes of pulse with pico - and femtosecond laser fluence with a wavelength $\lambda_{L}=0.8 \mu \mathrm{m}$ on two metal target of aluminum (Al) and copper $(\mathrm{Cu})$. The influence on each of the targets was carried out with the same energy density $F$, respectively $F=0.7 \mathrm{~J} \mathrm{~cm}^{-2}$ for $A l$ and $F=2.0 \mathrm{~J} \mathrm{~cm}^{-2}$ for $\mathrm{Cu}$.

Temperature dependences of the reflectivity $R\left(T_{e}\right)$ and volumetric absorption coefficient $\alpha\left(T_{e}\right)$ were calculated according to $[99,100]$. Fig. 14 shows the time profiles of the incident and the absorbed laser pulse with a Gaussian profile $\tau_{L}=10^{-12} \mathrm{~s}$ and $\tau_{L}=10^{-15} \mathrm{~s}$.

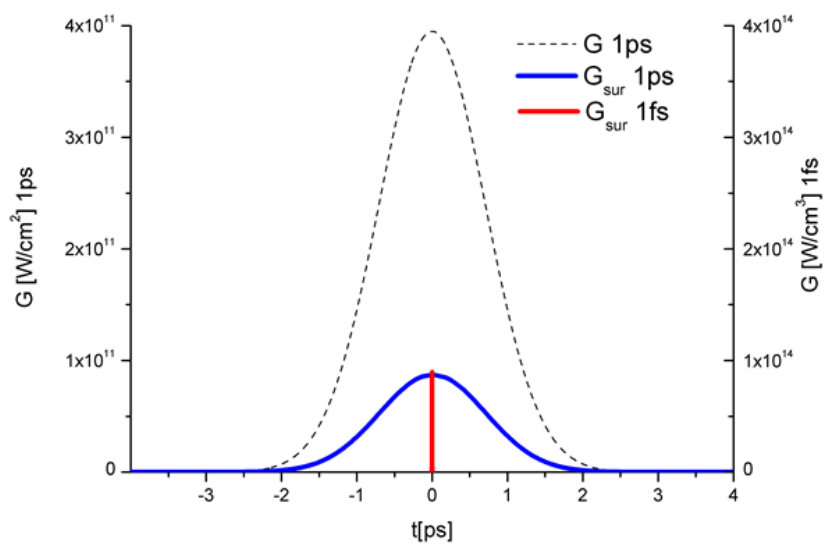

Figure 14. Time profile of pico- and femtosecond pulses on the surface of Al.

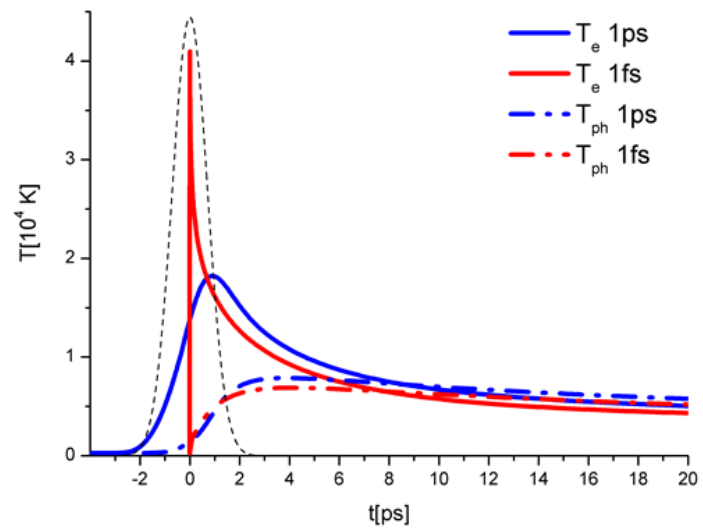

Figure 15. Time dependence of the surface temperature of Al.

The incident laser radiation is absorbed in the electron component, causing it to warm up fast. The main feature of the nonequilibrium heating is that it occurs under conditions of intense competition between the rapid release of the laser energy and the factors limiting the 
heat - strongly varying electron thermal conductivity coefficient $\lambda_{e}\left(T_{e}, T_{p h}\right)$ and electronfonon coupling factor $g\left(T_{e}\right)$.

At picosecond influence $\tau_{L}=10^{-12}$, the maximum of electron temperature of the surface $T_{e, \max } \approx 1.8 \cdot 10^{4} \mathrm{~K}$ is achieved at the descending branch of the laser pulse at the moment $t \cong \tau_{L}$, Fig. 15 , and the values of the coefficients vary in the range $\lambda_{e, \max } / \lambda_{A l}^{e q} \approx 10$ and $g_{\text {max }} / g_{A l}^{e q} \approx 70$. The maximum value of the lattice temperature is achieved after the pulse $t \approx 5 \tau_{L}$ and amounts $T_{p h \text { max }} \approx 7.5 \cdot 10^{3} \mathrm{~K}$. The maximum gap between the temperatures reaches the value $\Delta T=T_{e}-T_{p h} \approx 10^{4} \mathrm{~K}$. Temperature equalization $T_{e} \approx T_{p h}$ occurs within a time $\sim 10$ ps.

Femtosecond influence $\tau_{L}=10^{-15} \mathrm{~S}$ differs from picosecond that the same energy is released during the 3 orders of magnitude less than that causes more of a deviation from local thermodynamic equilibrium. The values of the coefficients $\lambda_{e}\left(T_{e}, T_{p h}\right)$ and $g\left(T_{e}\right)$ limiting the heating of electronic components, and also increase $\lambda_{e \text {, } \max } / \lambda_{A l}^{e q} \approx 27$ and $g_{\max } / g_{A l}^{e q} \approx 200$ , but not as much as $G(t)$. Time profile of the electron temperature of the surface $T_{e, \text { surf }}(t)$ significantly shifted relative to laser pulse time profile $G(t)$. The maximum value of the electron temperature is achieved at the end of the pulse $t \cong 2 \tau_{L}$ and is $T_{e \text {, } \max } \approx 4.1 \cdot 10^{4} \mathrm{~K}$, Fig. 15. A second difference of a femtosecond influence, despite the rapid energy exchange between the subsystems is a slow heating of the lattice, which differs little from the effects of heating at picosecond influence. The maximum value of the lattice temperature is achieved at the time $t \approx 5 \mathrm{ps}$ and is $T_{p h, \text { max }} \approx 7.2 \cdot 10^{3} \mathrm{~K}$. The maximum gap between the temperatures reaches a value $\Delta T=T_{e}-T_{p h} \approx 3.3 \cdot 10^{4} \mathrm{~K}$. Temperature equalization $T_{e} \approx T_{p h}$ occurs within the time $\sim 9$ ps.

The high heating rate determines high rate of phase transformations. Fig. 16 shows the time dependences of the melting front propagation velocity $v_{s l}(t)$ for two modes of influence. Since the rate of heating of the lattice by pico - and femtosecond pulses differ slightly, then the rate $v_{s l}$ is almost the same. The only difference is in the initial stage of the process. At the picosecond influence, the birth of the melting front starts at the front of the laser pulse $t \approx-0.1 p s$, while at the femtosecond with a considerable delay after the pulse, $t \approx 30 f_{s}$. The maximum values of $v_{s l \text { max }}(t) \approx 3.75 \mathrm{~km} / \mathrm{s}$ are achieved in the time interval $t \approx 2 \div 2.5 \mathrm{ps}$. Such a high rate of propagation of the front leads to a pressure jump at the melting surface of the solid phase $p_{s}=0.16 \mathrm{Mbar}$ for $\tau_{L}=10^{-12} \mathrm{~s}\left(p_{s}=0.14 \mathrm{Mbar}\right.$ for $\left.\tau_{L}=10^{-15} \mathrm{~s}\right)$ and the formation of a shock wave moving ahead of the front of melting, Fig. 17.

Appearance of high dynamic pressure causes an increase in the equilibrium melting temperature, the curve $T_{m}\left(p_{s}\right)$ shown in Fig. 18. Its maximum value reaches $T_{m}\left(p_{s}\right) \cong 1900 \mathrm{~K}$. Due to the dynamic pressure of about one order of magnitude as compared to the equilibrium value, increases the value of non-equilibrium heat of melting $L_{m}^{n e}\left(p_{s}\right)$.

Comparison of the $T_{s \ell}(t)$ and $T_{m}\left(p_{s}(t)\right)$ curves indicates a significant overheating of surface of the phase boundary $T_{s \ell}(t)>T_{m}\left(p_{s}(t)\right)$. The degree of nonequilibrium of melting process is conveniently characterized by the response function $\Delta T_{s \ell}\left(v_{s l}\right)$, which is shown in Fig.19. 


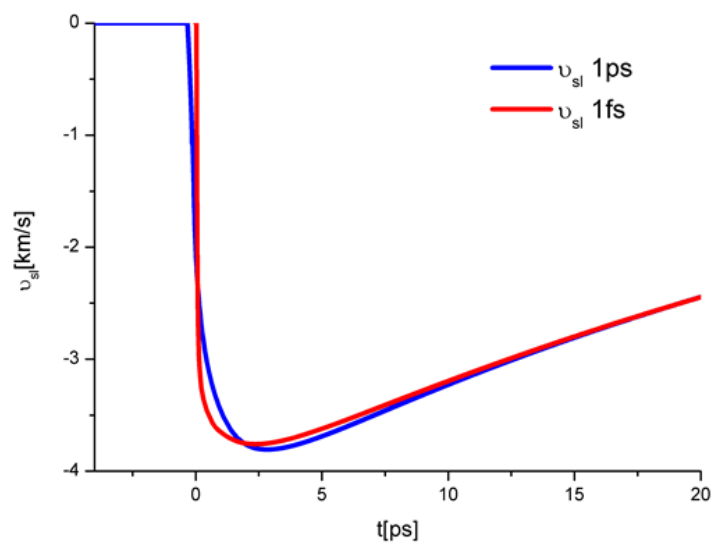

Figure 16. The time profile of the velocity of melting of aluminum.

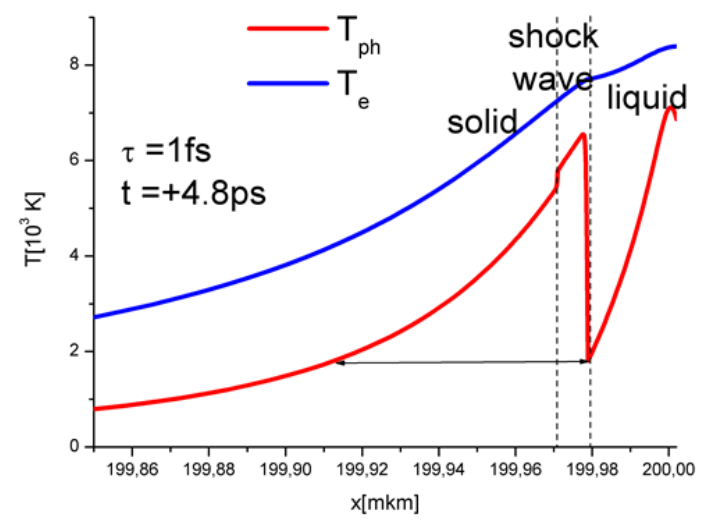

Figure 17. The spatial profiles of temperature of aluminum.

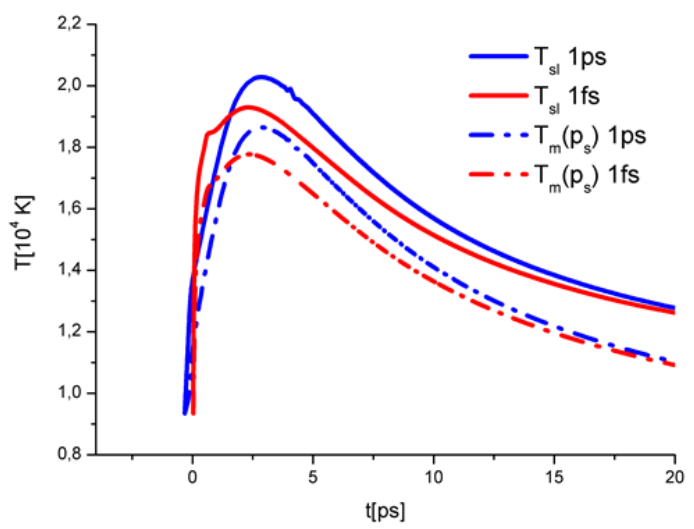

Figure 18. The time profiles of the equilibrium melting temperature and temperature of the melting front of Al. 


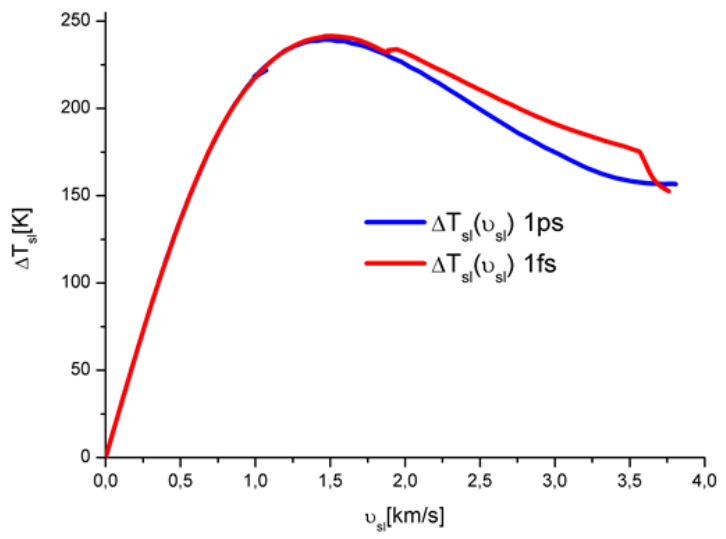

Figure 19. Response functions $\Delta T_{s \ell}\left(v_{s l}\right)$ for $\mathrm{Al}$.

At low overheating (supercooling), the function $\Delta T_{s \ell}$ increases linearly with growth $v_{s l}$. In the range of $v_{s l} \sim 1 \mathrm{~km} / \mathrm{s}$, at which the shock wave is formed in the solid phase, $\Delta T_{s \ell}$ function has a smooth maximum indicating the change of the mechanism of phase transformation. The thermal conductivity mechanism is dominant at low velocities $v_{s l} \leq 1 \mathrm{~km} / \mathrm{s}$ will be displaced by hydrodynamic at large values $v_{s l} \leq v_{\text {sound }}$.

Fig. 20 shows fragment of dependence of $v_{s l}(t)>0$ which characterizes the hardening process which takes place in the nanosecond time range. Maximum velocity of the solidification front does not exceed $45 \mathrm{~m} / \mathrm{s}$, respectively, the maximum undercooling is $10 \div 15 \mathrm{~K}$. High velocity of propagation of melting fronts are associated with strong crossflows of matter and energy across the phase boundary.

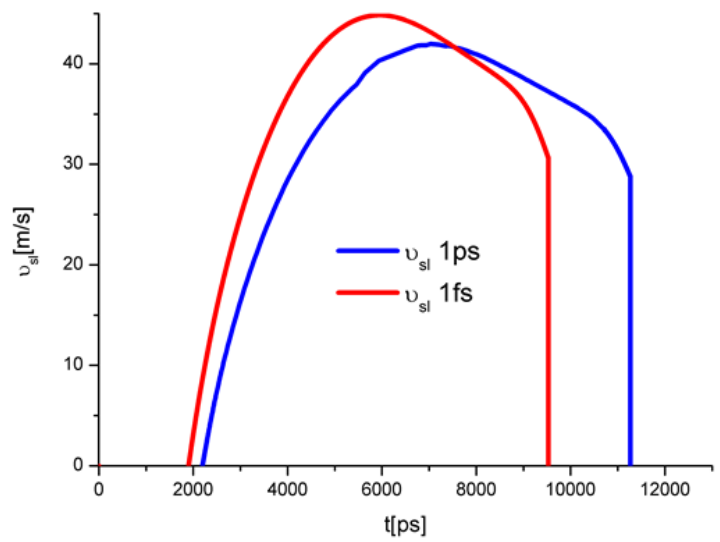

Figure 20. The time profiles of the velocity of solidification of aluminum.

In aggregate with volumetric nature of the energy transfer from the electron gas to the lattice it leads to the formation of highly superheated metastable state, characterized by the 
appearance of the surface temperature maximum $\Delta T_{p h \text {, max }}=T_{p h}-T_{m}\left(p_{s}\right) \approx 4.5 \cdot 10^{3} \mathrm{~K}$ in the solid phase, Fig. 17. In the liquid phase is also formed near-surface temperature maximum, Fig.20, but since the maximum velocity of evaporation front $v_{\ell v \text {, } \max } \approx 40 \mathrm{~m} / \mathrm{s}$ is two orders of magnitude lower than the velocity $v_{s l}$, magnitude and its role are insignificant.

Action of ultrashort laser pulses on metal target with different thermo-physical properties copper - has several differences and many in common with the action of the aluminum target. The main difference of the non-equilibrium heating and non-equilibrium phase transformation in the condensed media is in the first place connected with the rate of energy exchange between the electron and phonon subsystems. The coefficient of energy exchange $g\left(T_{e}\right)$ in copper is by about 1.5 orders lower than that of aluminum, so that during laser energy release, the action of one of the main factors that limit the heating of the electron subsystem turns out to be significantly lower. As a result, the maximum values of the electron temperature of the surface are several times higher than that for aluminum: $T_{e, \max } \approx 4 \cdot 10^{4} \mathrm{~K}$ for picosecond pulse $T_{e, \max } \approx 1.2 \cdot 10^{5} \mathrm{~K}$ for femtosecond pulse, fig. 21 .

Heating and melting of the lattice take place in the picosecond range $t_{m} \approx+0.1 p s$, fig.22. For copper, the rate of energy transfer to the lattice is significantly lower, so that the velocity of the melting front turns out to be several times lower than that for aluminum $v_{s \ell, \max }(t) \approx 1.3$ $\mathrm{km} / \mathrm{s}$, fig.23. The maximum value of the pressure in the solid phase is also lower, $p_{s, \max }=0.14 \mathrm{Mbar}$. Despite the fact that the velocity $v_{s \ell}$ for copper is significantly lower than that for $\mathrm{Al}$, the value of overheating of the interphase surface turns out to be significantly higher due to decrease of the hydrodynamic effects: $\Delta T_{s \ell, \max } \approx 350 \mathrm{~K}$, fig. 24 . The overheating of the sub-surface region of the solid phase reaches the value of $4 \cdot 10^{3} \mathrm{~K}$.

The solidification of the liquid phase takes place in the nanosecond rage - the same as for aluminum. The lifetime of the melt reaches the value of $15 \div 20 \mathrm{~ns}$, fig. 25 , the maximum velocity of the crystallization front $-v_{s \ell} \approx 35 \mathrm{~m} / \mathrm{s}$. The maximum value of the overcooling of the front $\sim 22 \mathrm{~K}$. The overcooling of the volume of the solid reaches the value of $800 \mathrm{~K}$.

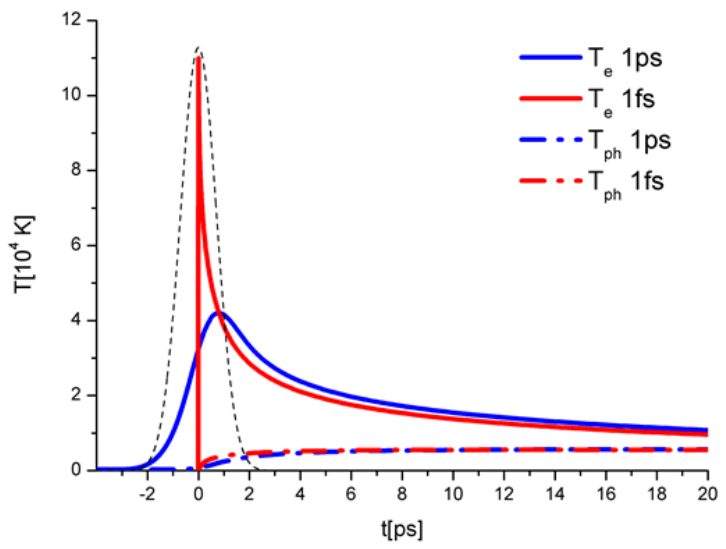

Figure 21. Time dependence of the surface temperature of $\mathrm{Cu}$. 


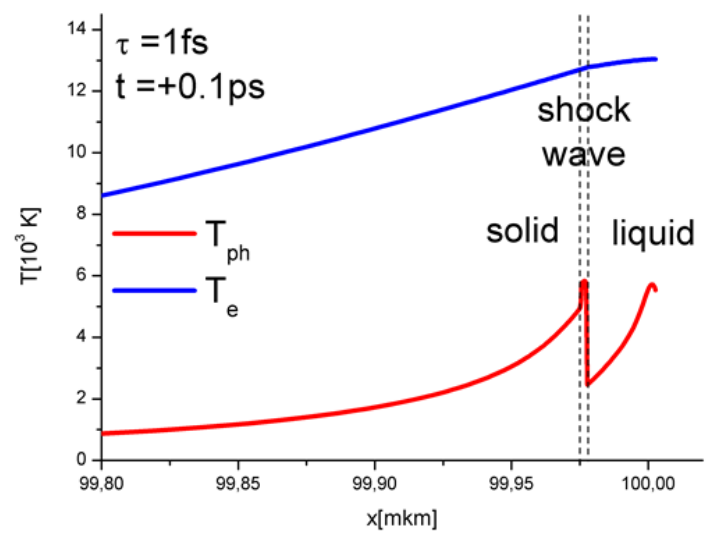

Figure 22. The spatial profiles of temperature of copper.

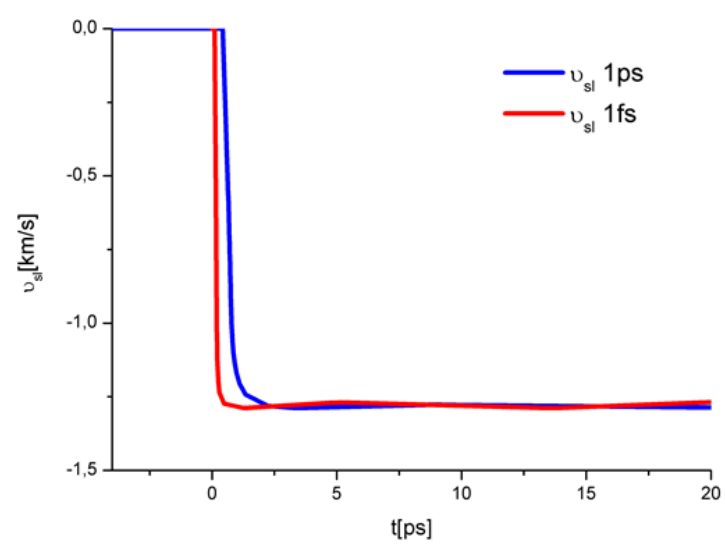

Figure 23. The time profile of the velocity of melting of copper.

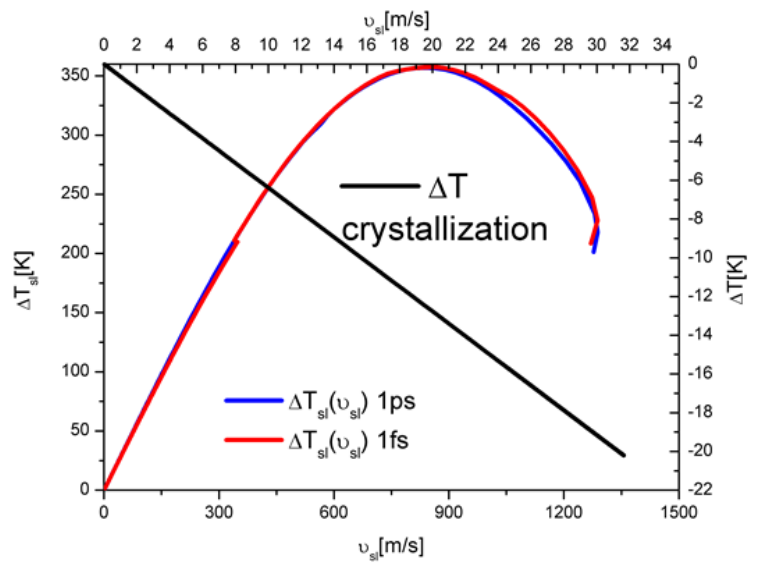

Figure 24. Response functions $\Delta T_{s \ell}\left(v_{s l}\right)$ for $\mathrm{Cu}$. 


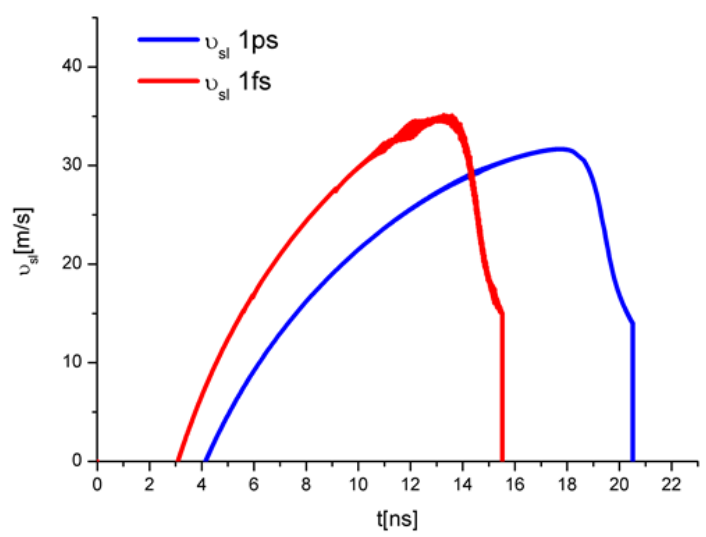

Figure 25. The time profiles of the velocity of solidification of copper.

Modern experimental methods of the investigation of the processes of meltingcrystallization of the metal systems determine the velocities of the solid-melt front to be within the range of $10 \div 100 \mathrm{~m} / \mathrm{s}$ with corresponding overcooling of liquid within the range of $10 \div 400 \mathrm{~K}$. Thus, the data from the modeling of the metastable overcooled states in the liquid phase of metals is in relatively good agreement with the experimental measurements. It is not possible to perform the same comparison with the overcooled states in the solid phase because of the lack of reliable experimental data which is caused by the complexity of statement of such experiments.

\section{Conclusion}

The detailed analysis of the physical processes of the non-equilibrium heating and the fast phase transformations in the metal systems under the action of ultra-short laser pulses was performed in this chapter. A mathematical model was built based on the brief analysis of the thermodynamical and kinetic approaches for the description of the phase transformations of the first order. This model is a non-equilibrium hydrodynamic version of the Stefan problem and describes the kinetics of high-speed heterogeneous phase transformations: melting, crystallization, evaporation. The model is supplemented by the explicit description of the moving phase fronts for correct reflection of the dynamics of the phase transitions.

The methods of mathematical modeling were used to investigate the regimes of pico- and femtosecond action on metals - $\mathrm{Al}, \mathrm{Cu}$. The kinetics and dynamics of phase transformations are analyzed. The results of the modeling confirmed the skewness of the high-speed processes of melting - solidification. In particular, the maximum velocity of melting reaches the value of several kilometers per second, while the maximum velocity of solidification does not exceed hundred of meters per second. The temperature dependence $v_{s \ell}\left(T_{s \ell}\right)$ does not have a sharp bend in the point of change of the direction of the phase transition. 
Special attention is paid to the overheated and overcooled metastable states that are caused by the fast phase transitions. The deviation from the local thermodynamical equilibrium is noticed both in the bulk and at the interphase boundary. The response function $T_{s \ell}\left(v_{s \ell}\right)$ has a linearly increasing character for low values of velocity $v_{s \ell}$ in the range of $[0 \div \pm 50] \mathrm{m} / \mathrm{s}$. As the velocity $v_{s \ell}$ rises more than $v_{s \ell}>100 \mathrm{~m} / \mathrm{s}$, a maximum is formed in the response function for both metals. The appearance of the maximum means that the dominating mechanism of the phase transition changes: the mechanism of heat conductivity is changed by the hydrodynamic one. The maximum value of overheating of the interphase boundary reaches the value of $200 \div 400 \mathrm{~K}$, the one for overcooling is by one order lower than that value. The overheating and overcooling of the bulk are significantly higher $4 \cdot 10^{3} \div 5 \cdot 10^{3} \mathrm{~K}$ for overheating and $6 \cdot 10^{2} \div 8 \cdot 10^{2} \mathrm{~K}$ for overcooling. The results for different materials are compared between each other and with experimental data.

These results do not pretend to be an exhaustive description of the variety of fast phase transitions, because they used the assumption of the thermodynamic stability of overheated / overcooled metastable states during all times considered. Refusal of such assumptions will require further research to include the kinetics of homogeneous phase transformations into the consideration.

\section{Author details}

V.I. Mazhukin

Keldysh Institute of Applied Mathematics of RAS, Moscow, Russia

\section{Acknowledgement}

This work was supported by the RFBR (projects nos. 10-07-00246-a, 12-07- 00436-a, 11-0112086-ofi-m).

\section{References}

[1] Fujimoto J.G., Liu J.M., Ippen E.P., Bloembergen N (1984) Femtosecond Laser Interaction with Metallic Tungsten and Nonequilibrium Electron and Lattice Temperatures. Physical Review Letters. 53: 19/1837 - 1840.

[2] Chichkov B.N., Momma C., Nolte S., von Alvensleben F, Tunnermann A. (1996) Femtosecond, picosecond and nanosecond laser ablation of solids. Appl. Phys. A 63: 109-115.

[3] Shirk M.D., Molian P.A. (1998) A review of ultrashort pulsed laser ablation of materials. Journal of Laser Applications. 10: 1/18-28.

[4] Von der Linde D., Sokolovski-Tinten K. (2000) The physical mechanisms of short-pulse laser ablation. Appl. Surf. Sci. 154-155: 1-10. 
[5] (2007) Part 2: Ultrafast Interactions. In: Claude R. Phipps, editor. Optical Sciences. Springer Series in Laser ablation and its applications. Santa Fe, New Mexico: Springer. 129: 121-280.

[6] Gonzales P., Bernath R., Duncan J., Olmstead T., Richardson M. (2004) Femtosecond ablation scaling for different materials. Proc. SPIE. 5458: 265-272.

[7] Chichkov B.N., Korte F., Koch J., Nolte S., Ostendorf A. (2002) Femtosecond laser ablation and nanostructuring. Proc. SPIE. 4760: 19-27.

[8] Hamad S., Krishna Podagatlapalli G., Sreedhar S., Tewari S. P., Rao S. V. (2012) Femtosecond and picosecond ablation of aluminum for synthesis of nanoparticles and nanostructures and their optical characterization. Proc. SPIE 8245: 82450L.

[9] Ostendorf A., Kamlage G., Klug U., Korte F., Chichkov B. N. (2005) Femtosecond versus picosecond laser ablation. Proc. SPIE 5713:1- 8.

[10] Kamlage G., Bauer T., Ostendorf A., Chichkov B.N. (2003) Deep drilling of metals by femtosecond laser pulses. Applied Physics A: Materials Science \& Processing. 77: 307310.

[11] Lopez J., Kling R., Torres R., Lidolff A., Delaigue M., Ricaud S., Hönninger C., Mottay E. (2012) Comparison of picosecond and femtosecond laser ablation for surface engraving of metals and semiconductors. Proc. SPIE 8243: 82430O.

[12] Bulgakov A.V., Bulgakova N. M. Burakov I.M. (2009) Nanosized Material Synthesis by Action of High - Power Energy Fluxes on Matter. Novosibirsk: Institute Thermophysics SB RAS. 461 p.

[13] Mazhukin V.I., Lobok M.G., Chichkov B.N. (2009) Modeling of fast phase transitions dynamics in metal target irradiated by pico and femto second pulsed laser. Applied Surface Science. 255: 5112-5115.

[14] Herlach D.M., Galenko P.K., Holland-Moriz D. (2005) Metastable materials from undercooled metallic melts. Amsterdam: Elsevier. 485 p.

[15] Eliezer S., Eliaz N., Grossman E., Fisher D., Gouzman I., Henis Z., Pecke S., Horovitz Y., Fraenkel M., Maman S., Lereah Y. (2004) Synthesis of nanoparticles with femtosecond laser pulses. Phys. Rev. B 69: 144119/1-6.

[16] Mene'ndez-Manjo'n A., Barcikowski S., Shafeev G.A., Mazhukin V.I., Chichkov B.N. (2010) Influence of beam intensity profile on the aerodynamic particle size distributions generated by femtosecond laser ablation. Laser and Particle Beams. 28: 45-52.

[17] Stratakis E., Barberoglou M., Fotakis C., Viau G., Garcia C., Shafeev G. A. (2009) Generation of $\mathrm{Al}$ nanoparticles via ablation of bulk $\mathrm{Al}$ in liquids with short laser pulses. Opt. Express. 17: 12650-12659.

[18] Mazhukin V.I., Samarskii A.A. (1994) Mathematical Modeling in the Technology of Laser Treatments of Materials. Review. Surveys on Mathematics for Industry. 4: 2/85149.

[19] Ivanov D.S., Zhigilei L.V. (2003) Combined atomistic-continuum modeling of shortpulse laser melting and disintegration of metal films. Phys. Rev. B. 68: 064114-1 064114-22.

[20] . Landau L. D., Lifshic E. M. (1976) Teoreticheskaja fizika, t. V. Statisticheskaja fizika. Chast' 1. M.: Nauka. 616 p. 
[21] Christian J. W. (1965) The theory of transformations in metals and alloys: an advanced textbook in physical metallurgy. Oxford: Pergamon Press. 975 p.

[22] Kirkpatrick R. J. (1975) Crystal Growth from the Melt. Review. American Mineralogist A: 60: 798-614.

[23] Asta M., Beckermann C., Karma A., Kurz W., Napolitano R., Plapp M., Purdy G., Rappaz M., Trivedi R. (2009) Solidification microstructures and solid-state parallels: Recent developments, future directions. Acta Materialia: 57: 146/941-971.

[24] Jackson K. A. (2004) Kinetic Processes:Crystal Growth, Diffusion, and Phase Transitions in Materials. Weinheim: Wiley-VCH Verlag GmbH \& Co. KGaA. 409 p.

[25] Hoyt J. J., Asta M. (2002) Atomistic computation of liquid diffusivity, solid-liquid interfacial free energy, and kinetic coefficient in Au and Ag. Phys. Rev. B. 65: 21/2141061 - 214106-11.

[26] Rodway G.H., Hunt J.D. (1991) Thermoelectric investigation of solidification of lead I. Pure lead. Journal of Crystal Growth. 112: 2-3/554-562.

[27] Hoyt J.J., Asta M., Karma A. (2002) Atomistic Simulation Methods for Computing the Kinetic Coefficient in Solid-Liquid Systems. Interface Science. 10: 2-3/181-189.

[28] Buta D., Asta M., Hoyt J. J. (2007) Kinetic coefficient of steps at the Si(111) crystal-melt interface from molecular dynamics simulations. Journal of Chemical Physics. 127: 7/074703 - 074713 .

[29] Amini M., Laird B. B. (2006) Kinetic Coefficient for Hard-Sphere Crystal Growth from the Melt. Physical Review Letters. 97: 216102-1 - 216102-4.

[30] Monk J., Yang Y., Mendelev M. I., Asta M., Hoyt J. J., Sun D. Y. (2010) Determination of the crystal-melt interface kinetic coefficient from molecular dynamics simulations. Modelling and Simulation in Materials Science and Engineering. 18: 015004-1 - 01500418.

[31] Wilson H.A. (1900) On the velocity of solidification and viscosity of supercooled liquids. Philos. Mag. 50: 238-250.

[32] Frenkel Ja. I. (1932) Note on the relation between the speed of crystallization and viscosity. Phys. Z. Sowjet Union. 1: $498-499$.

[33] Frenkel' Ja.I. (1975) Kineticheskaja teorija zhidkostei. L.: Nauka. 592 p.

[34] Jackson K.A., Chalmers B. (1956) Kinetics of solidification. Can. J. Phys. 34: 473 - 490.

[35] Chalmers B. (1964) Principles of Solidification., New York: John Wiley \&Sons. 129 p.

[36] Klude M. D., Ray J.R. (1989) Velocity versus Temperature Relation for Solidification and Melting of Silicon: A Molecular-Dynamics Study. Phys. Rev. B. 39: 3/1738-1746.

[37] Tymczak C.J. Ray J.R. (1990) Interface response function for a model of sodium: A molecular dynamics study, J. Chem. Phys. 92: 12/7520 - 7530.

[38] Tymczak C.J. Ray J.R. (1990), Asymmetric crystallization and melting kinetics in sodium: A molecular-dynamics study. Phys. Rev. Lett. Vol. 64, Issue 11, pp. 1278-1281.

[39] MacDonald C. A., Malvezzi A. M., Spaepen F. (1989) Picosecond time-resolved measurements of crystallization in noble metals. J. Appl. Phys. 65: 1/129-137.

[40] Hertz H. (1882) Uber die Verdunstung der Flussigkeiten, insbesondere des Quecksilbers, im luftleeren Raume. Ann. Phys. und Chemie. 17: 177-190. 
[41] Knudsen M. (1915) Die Maximale Verdampfungs-geschwindigkeit Quecksilbers. Ann. Phys. und Chemie. 47: 697-705.

[42] Siewert C.E., Thomas J.R. (1981) Strong evaporation into a half space. J. of Applied Mathem. and Physics( ZAMP). 32: 421 - 433.

[43] Siewert C.E. (2003) Heat transfer and evaporation/condensation problems based on the linearized Boltzmann equation. European Journal of Mechanics B/Fluids. 22: 391-408.

[44] Frezzotti A. (2007) A numerical investigation of the steady evaporation of a polyatomic gas. European Journal of Mechanics B/Fluids. 26: 93-104.

[45] Titarev V. A., Shakhov E. M. (2001) Teplootdacha i isparenie s ploskoi poverkhnosti v poluprostranstvo pri vnezapnom povyshenii temperatury tela. Mekhanika zhidkosti i gaza. 1/141 - 153 .

[46] Abramov A. A., Butkovsky A. V. (2009) Effect of the Mode of Gas-Surface Interaction on Intensive Monatomic Gas Evaporation. Fluid Dynamics. 44: 1/80-87.

[47] Bond M., Struchtrup H. (2004) Mean evaporation and condensation coefficients based on energy dependent condensation probability. Phys. Rev. E. 70: 061605-1 - 20.

[48] Ford I J, Lee T-L. (2001) Entropy production and destruction in models of material evaporation. J. Phys. D: Appl. Phys. 34: 413-417.

[49] Rose J. W. (2000) Accurate approximate equations for intensive sub-sonic evaporation. Int. Journal of Heat and Mass Transfer.. 43: 3869 - 3875.

[50] Bedeaux D., Kjelstrup S., Rubi J. M. (2003) Nonequilibrium translational effects in evaporation and condensation. J. Chemical Physics. 119: 17/9163 - 9170.

[51] Frezzotti A., Gibelli L., Lorenzani S. (2005) Mean field kinetic theory description of evaporation of a fluid into vacuum. Phys. Fluids. 17: 012102-1 - 012102-12.

[52] Rahimi P., Ward Ch. A. (2005) Kinetics of Evaporation: Statistical Rate Theory Approach Int.J. of Thermodynamics. 8: 1/1-14.

[53] Meland R., Frezzotti A., Ytrehus T., Hafskjold B. (2004) Nonequilibrium moleculardynamics simulation of net evaporation and net condensation, and evaluation of the gas-kinetic boundary condition at the interphase. Phys. Fluids. 16: 2/223 - 243.

[54] Ishiyama T., Yano T., Fujikawa S. (2005) Kinetic boundary condition at a vapor-liquid interface. Phys. Rev. Lett. 95: 0847504.

[55] Frezzotti A. (2011) Boundary conditions at the vapor-liquid interface. Phys. Fluids. 23: 030609-(1-9).

[56] Kartashov I.N., Samokhin A.A., Smurov I.Yu. (2005) Boundary conditions and evaporation front instabilities. J. Phys. D: Appl. Phys. 38: 3703-3714.

[57] Crout D. (1936) An application of kinetic theory to the problem of evaporation and sublimation of monatomic gases. J. Math. Physics. 15: 1-54.

[58] Labuntsov D.A., Kryukov A.P. (1979) Analysis of Intensive Evaporation and Condensation. Inter. J. Heat and Mass Transfer. 22: 7/989-1002.

[59] Mazhukin V.I., Samokhin A.A. (1987) Matematicheskoe modelirovanie fazovykh perekhodov i obrazovanija plazmy pri deistvii lazernogo izluchenija na pogloshhajushhie sredy. In: Samarskii A.A., Kurdjumov S.P., Mazhukin V.I., editors. Nelineinye differencial'nye uravnenija matematicheskoi fiziki. Moskva: Nauka. pp. 191244. 
[60] Samokhin A.A. (1990) Effect of laser radiation on absorbing condensed matter. In: Prokhorov A.M. Series editor. Fedorov V.B. editor. Proceedings of the Institute of General Physics Academy of Sciences of the USSR. New-York: Nova Science Publishers. 13: 203 p.

[61] Anisimov S.I. (1968) Ob isparenii metalla, pogloshhajushhego lazernoe izluchenie. ZhEhTF. 54: 1/339-342.

[62] Khight C.J. (1979) Theoretical Modeling of Rapid Surfact Vaporation with Back Pressure. AIAA J. 17: 5/81-86.

[63] Mazhukin V.I., Prudkovskii P.A., Samokhin A.A. (1993) O gazodinamicheskikh granichnykh uslovijakh na fronte isparenija. Matematicheskoe modelirovanie. 5: 6/3-9.

[64] Mazhukin V.I., Samokhin A.A. (2012) Boundary conditions for gas-dynamical modeling of evaporation processes. Mathematica Montisnigri. XXIV: 8-17.

[65] Lifshic I.M., Kaganov M.I., Tanatarov L.V. (1956) Relaksacija mezhdu ehlektronami i kristallicheskojj reshetkojj. ZhEhTF. 31: 2(8)/232-237.

[66] Lifshic I.M., Kaganov M.I., Tanatarov L.V. (1959) K teorii radiacionnykh izmenenijj v metallakh. Atomnaja ehnergija. 6/391-402.

[67] Martynenko Ju.V., Javlinskii Ju.N. (1983) Okhlazhdenie ehlektronnogo gaza metalla pri vysokojj temperature. DAN SSSR. 270: 1/88-91.

[68] Beer A.C., Chase M.N., Choquard P.F. (1955) Extension of McDougall-Stoner tables of the Fermi-Dirac functions. Helvetica Physica Acta. XXVII: 5/529-542.

[69] Kittel C. (1978) Introduction to Solid State Physics, 6h Edition. New York: J. Wiley \& Sons. 789 p.

[70] Landau L.D., Lifshic E.M. (1963) Teoreticheskaja fizika. Kvantovaja mekhanika. Nereljativistskaja teorija. T.III. Moskva: Fizmatgiz. 702 p.

[71] Frenkel' Ja.I. (1958) Vvedenie v teoriju metallov. Moskva: Fizmatlit. 368 p.

[72] Touloukian Y. S., Powell R. W., Ho C. Y. and Klemens P. G (1970) Thermophysical Properties of Matter Volume 1: Thermal Conductivity: Metallic Elements and Alloys, New York, Washington: IFI/Plenum Data Corp.

[73] Ziman J.M (1974) Principles of the theory of solid. Gambridge: University Press. 472 p.

[74] Cheynet, B., J.D. Dubois, M. Milesi (1996) Données thermodynamiques des éléments chimiques. In : Techniques de L'Ingenieur, Materiaux metalliques. No. M153. Forum M64. pp. 1-22

[75] Aluminum: Properties and physical metallurgy (1984) In: John E. Hatch editor. American society for metals. Ohio: Metals Park. 421 p.

[76] Fizicheskie velichiny. Spravochnik (1991) Grigor'eva I.S., Mejjlikhova E.Z. editors. Moskva: Ehnergoatomizdat. 1232 p.

[77] Iida T., Guthrie R.I.L. (1988) The Physical Properties of Liquid Metals. Oxford: Clarendon Press. 288 p.

[78] Pines D (1965) Ehlementarnye vozbuzhdenija v tverdykh telakh. Moskva: Mir. 383 p.

[79] Qiu T.Q., Tien C.L (1994) Femtosecond Laser Heating of Multi-Layer Metals. I. Analysis. Int. J. Heat Mass Transfer. 37: 17/2789-2797.

[80] Ginzburg V.L., Shabanskii V.L (1955) Kineticheskaja temperatura ehlektronov v metallakh i anomal'naja ehlektronnaja ehmissija. Dokl. AN SSSR. 100: 3/445 - 448. 
[81] Martynenko Ju.V., Javlinskii Ju.N (1987) Vozbuzhdenie ehlektronov metalla oskolkom delenija. Atomnaja ehnergija. 62: 2/80-83.

[82] Lin Z., Zhigilei L.V., Celli V (2008) Electron-phonon coupling and electron heat capacity of metals under conditions of strong electron-phonon nonequilibrium. Phys. Rev. B. 77: 7/075133-1 - 075133-17.

[83] Mazhukin V.I., Mazhukin A.V., Demin M.M., Shapranov A.V. (2011) Ehffekty neravnovesnosti pri vozdejjstvii impul'snogo lazernogo izluchenija na metally. Opticheskiy zhurnal. 78: 8/29-37.

[84] Mazhukin V.I., Mazhukin A.V., Demin M.M., Shapranov A.V. (2011) Mathematical modeling of short and ultrashort laser action on metals. In: Sudarshan T.S., Beyer Eckhard, Berger Lutz-Michael editors. Surface Modification Technologies (STM 24). Dresden: Fraunhofer Institute for Material and Beam Technology IWS. 24: 201 -208.

[85] Samarskii A.A., Moiseenko B.D (1965) Ehkonomichnaja skhema skvoznogo scheta dlja mnogomernojj zadachi Stefana. ZhVM i MF. 5: 5/816-827.

[86] Meyer G.H (1973) Multidimensional Stefan Problems. SIAM J. Numer. Anal. 10: 3/522538.

[87] White B.R.E (1983) A modified finite difference scheme for the Stefan problem. Mathem. of Comput. 41: 164/337-347.

[88] Klimontovich Ju.L (1982) Statisticheskaja fizika. Moskva: Nauka. 608 p.

[89] Mazhukin V.I., Smurov I., Flamant G (1995) Overheated Metastable States in Pulsed Laser Action on Ceramics. J. Applied Physics. 78: 2/1259-1270.

[90] Mazhukin V.I., Smurov I., Dupuy C., Jeandel D (1994) Simulation of Laser Induced Melting and Evaporation Processes in Superconducting. J. Numerical Heat Transfer Part A. 26: 587-600.

[91] V.I.Mazhukin, M.G.Lobok, B.N.Chichkov (2009) Modeling of fast phase transitions dynamics in metal target irradiated by pico and femto second pulsed laser. Applied Surface Science. 255/5112-5115.

[92] Mazhukin V.I., Samarskii A.A., Chujjko M.M (1999) Metod dinamicheskojj adaptacii dlja chislennogo reshenija nestacionarnykh mnogomernykh zadach Stefana. Doklady RAN. 368: 3/307 - 310.

[93] Mazhukin A.V., Mazhukin V.I (2007) Dinamicheskaja adaptacija v parabolicheskikh uravnenijakh. Zhurnal vychislitel'nojj matematiki i matematicheskojj fiziki. 47: 11/1911 1934. Mazhukin A.V., Mazhukin V.I (2007) Dynamic Adaptation for Parabolic Equations. Computational Mathematics and Mathematical Physics. 47: 11/1833 - 1855.

[94] Breslavsky P.V., Mazhukin V.I (2008) Metod dinamicheskojj adaptacii v zadachakh gazovojj dinamiki s nelinejjnojj teploprovodnost'ju. Zhurnal vychislitel'nojj matematiki i matematicheskojj fiziki. 48: 11/2067-2080. Breslavskii P. V. and Mazhukin V. I (2008) Dynamic Adaptation Method in Gasdynamic Simulations with Nonlinear Heat Conduction. Computational Mathematics and Mathematical Physics. 48: 11/2102-2115.

[95] Koroleva O.N., Mazhukin V.I (2006) Matematicheskoe modelirovanie lazernogo plavlenija i isparenija mnogoslojjnykh materialov. Zhurnal vychislitel'nojj matematiki i matematicheskojj fiziki. 46: 5/910 - 925. Koroleva O.N., Mazhukin V.I (2006) 
Mathematical Simulation of Laser Induced Melting and Evaporation of Multilayer Materials. Computational Mathematics and Mathematical Physics. 46: 5/848 - 862.

[96] Mazhukin V.I., Samarskii A.A., Kastel'janos Oplando, Shapranov A.V (1993) Metod dinamicheskojj adaptacii dlja nestacionarnykh zadach's bol'shimi gradientami. Matematicheskoe modelipovanie. 5: 4/32-56.

[97] Samarskii A.A (1989) Teorija raznostnykh skhem. Moskva: Nauka. 616 p.

[98] Samarskii A.A., Popov Ju.P (1992) Raznostnye metody reshenija zadach gazovojj dinamiki. Moskva: Nauka. 421pp.

[99] Mazhukin V.I., Mazhukin A.V., Koroleva O.N (2009) Optical properties of electron Fermi-gas of metals at arbitrary temperature and frequency. Laser Physics. 19: 5/1179 1186.

[100] Mazhukin A.V., Koroleva O.N (2010) Raschjot opticheskikh kharakteristik aljuminija. Matematicheskoe modelirovanie. 22: 5/15-28. 